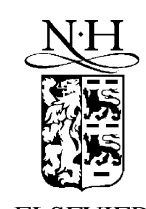

Mathematics and Computers in Simulation 51 (1999) 63-90

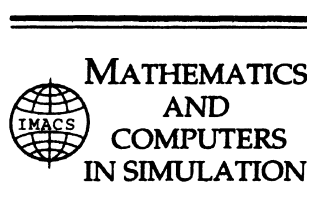

www.elsevier.nl/locate/matcom

\title{
Multistability in a dynamic Cournot game with three oligopolists
}

\author{
Hamdy Nabih Agiza ${ }^{\mathrm{a}, 1}$, Gian Italo Bischi ${ }^{\mathrm{b}, *}$, Michael Kopel ${ }^{\mathrm{c}, 2}$ \\ ${ }^{a}$ Mathematics Department, Faculty of Science, Mansoura University, Mansoura 35516, Egypt \\ ${ }^{\mathrm{b}}$ Istituto di Scienze Economiche, University of Urbino 61029, Urbino, Italy \\ ${ }^{\mathrm{c}}$ Department of Managerial Economics and Industrial Organization, \\ University of Technology Theresianumgasse 27, 1040 Vienna, Austria
}

Received 6 May 1999; accepted 14 June 1999

\begin{abstract}
The time evolution of a dynamic oligopoly game with three competing firms is modeled by a discrete dynamical system obtained by the iteration of a three-dimensional non-invertible map. For the symmetric case of identical players a complete analytical study of the stability conditions for the fixed points, which are Nash equilibria of the game, is given. For the situation of several coexisting stable Nash equilibria a numerical study of their basins of attraction is provided. This gives, evidence of the occurrence of global bifurcations at which the basins are transformed from simply connected sets into nonconnected sets, a basin structure which is peculiar of non-invertible maps. The presence of several coexisting attractors (or multistability) is observed even when complex attractors exist. Two different routes to complexity are presented: one related to the creation of more and more complex attractors; the other related to the creation of more and more complex structures of the basins. Starting from the benchmark case of identical players, the effects of heterogeneous behavior of the players, causing the loss of the symmetry properties of the dynamical system, are investigated through numerical explorations. (C) 1999 IMACS/ Elsevier Science B.V. All rights reserved.
\end{abstract}

Keywords: Discrete dynamical systems; Duopoly games; Non-invertible maps; Basins of attraction; Bifurcations

\section{Introduction}

About 160 years ago Cournot [9] introduced a model of imperfect competition between firms, and by now it has become a central concept in the field of industrial organization. The Cournot model analyzes a situation of an oligopoly, a market structure between monopoly and perfect competition. In an oligopoly there are only few firms in the market, and strategic considerations play an important role:

\footnotetext{
* Corresponding author.

E-mail address: bischi@econ.uniurb.it (G. Italo Bischi)

${ }^{1}$ E-mail address: agizah@mum.mans.eun.eg (H.N. Agiza)

${ }^{2}$ E-mail address: kopel@ebwnov.tuwien.ac.at (G.I. Bischi)
} 
each of the competitors tries to outguess what the other firms will do in order to gain a comparative advantage over the other firms, i.e. they form expectations concerning how their rivals will act. The expectations on the competitor's actions can be used to calculate the firm's own most preferred action, which is the profit-maximizing choice given these expectations ${ }^{3}$. For economists one constellation is of particular interest, namely a situation where all actions and expectations are consistent, i.e. where the expected and the actually taken choices coincide. Such a situation can be characterized by the fact that no firm has an incentive to unilaterally deviate from it's chosen strategy given the (expected) choices of it's rivals, and it is referred to as Cournot-Nash-Equilibrium in the economics literature ${ }^{4}$. Such a Nash equilibrium might then serve as a prediction of what outcome will be observed in an oligopoly market.

However, it seems unlikely that firms would immediately coordinate on such an equilibrium. Accordingly, economists started to ask if competitors would learn to play according to a Nash equilibrium profile over time, and this led them to an analysis of the stability properties of the emerging Nash equilibria and to the consideration of various dynamic adjustment processes. In the original work of Cournot, as well as in many subsequent papers, the reaction functions of the firms are decreasing (often linear) functions, and their unique intersection point gives the unique Nash equilibrium (see [5] for references). In such a case, the equilibrium in the oligopoly model has simple stability properties for a broad range of adjustment processes as the trajectories can either converge to the Nash equilibrium or diverge.

In this paper we consider a Cournot oligopoly game with three competitors ${ }^{5}$. Our model generalizes the duopoly model which was introduced in [16] and recently studied in [2,5,6]. In contrast to various existing studies, in these papers it is assumed that the optimal actions of the duopolists are characterized by unimodal reaction curves. Non-monotonic reaction functions arise quite naturally in several economic contexts: they might be due to externalities in the cost functions, competitors might regard their products as strategic complements, or the market can be described by a demand function with constant elasticity. In order to clarify the economic meaning of such sources of non-monotonicities the following examples may be helpful: interfirm externalities in the cost function capture the fact that a firm's costs might depend on the actions of the competitors. Consider, for example, a firm which sells books. This firm might profit from the actions of other book sellers, since customers develop a habit of buying books as they become more aware of them. Unimodal reaction curves arise since the sales of one firm will be best when the other firms sell a positive but moderate amount. A similar example might serve to illustrate a situation where products are strategic complements: the (marginal) profit of one firm increases with the competitors' actions. A Cournot game with three oligopolists and unimodal reaction functions has been recently studied by Puu [21]. Also in this case, the model extends a duopoly model proposed by the same author in [19]. The unimodal reaction functions in these papers are obtained by assuming a non-linear (isoelastic) demand function and constant marginal costs. The same demand and cost functions have been considered in [3], where an extension of the study of local

\footnotetext{
${ }^{3}$ The decision rule which describes the profit-maximizing actions of a firm given the expectations about the rival's choices is referred to as \% reaction function in what follows.

${ }^{4} \mathrm{~A}$ Nash Equilibrium is a profile of strategies such that each firm's strategy is an optimal response to the other firms' strategies. In the Nash equilibrium none of the firms has an incentive to deviate, since each firm's strategy is that firm's best response to the other firms' predicted strategies. The equilibria are simply the profiles given by the intersection points of the reaction functions of the oligopolists.

${ }^{5}$ An oligopoly with two firms is often called a duopoly and an oligopoly with three firms is sometimes referred to as a triopoly.
} 
stability to the case of $n$ competitors is given. Our paper differs, however, from these studies: whereas the models analyzed there give rise to a unique Nash equilibrium, the dynamic Cournot game we consider is characterized by the presence of several Nash equilibria. In fact, in our model up to eight Nash equilibria may exist, and several of them (up to four) can be simultaneously stable. This leads to two somehow related questions, which are of considerable importance for economists as well as mathematicians: first, which of the Nash equilibria (fixed points) will be selected in the long run? Second, which role do the initial actions chosen by the firms play in determining the long run outcome of the market? The main focus of this paper is to give an answer to these questions.

Due to the coexistence of several stable Nash equilibria, an answer requires not only a study of the stability regions in the space of the model's parameters, but also a study of the basins of attraction in the phase space, in order to ascertain the role of the starting conditions of the game in the selection of the final outcome. The study of the general Cournot game with three heterogeneous players is not an easy task, however, since no analytic expression can be obtained for the fixed points, as well as for their stability conditions. For this reason, we make an assumption which is often found in the literature to simplify the analysis: the present paper is mainly devoted to the study of the particular case of three identical (or homogeneous) oligopolists. Under this assumption the dynamical system which describes the time evolution of the repeated game has reflectional as well as rotational symmetries. In this case it is possible to obtain the analytical expressions of the fixed points (Nash equilibria of the Cournot game) and of the conditions for their local bifurcations. The symmetry properties imply that coexisting stable Nash equilibria, as well as more complex coexisting attractors, might be present. Moreover, in order to show that the property of coexistence of attracting sets is robust - in the sense that they also exist after the introduction of small heterogeneities - we also present numerical simulations for the case of heterogeneous players. The results suggest that the particular case of identical players constitutes a useful benchmark case for the understanding of the more general (and realistic) case of oligopolists exhibiting heterogeneous behavior.

The aim of our paper is two-fold. From an economic point of view, the paper deals with an extension of a Cournot duopoly game to the case of three competitors. Typical questions which arise in the study of competition models (actually not only in the context of economic systems, but also in a larger class of models, such as those studied, e.g., in ecology) are: what is the effect of introducing a new competitor? Is the system more stable or more vulnerable? Are the dynamics more complex? Our analysis will point out analogies and differences between the duopoly and the triopoly case. We shall not only consider the question of local stability, but also the problem of the delimitation of the basins of attraction, at least by numerical methods.

The second goal of the paper concerns the mathematical properties of the proposed model. In fact, we give a fairly complete study of a three-dimensional discrete-time dynamical system which exhibits rather complex behavior. This is due to both the creation of complex attractors in the three-dimensional phase space and to the coexistence of attracting sets with rather complicated basin boundaries. It should be pointed out here that in the last 20 years a good level of understanding of one-dimensional noninvertible discrete dynamical systems has been reached. Furthermore, some important insights have been obtained in the analysis of two-dimensional non-invertible maps, see $[1,14,17]$. These results have been recently applied to the study of Cournot duopoly games (see [4,5,7,22]). However, the mathematical treatment of three-dimensional non-invertible maps, like the one considered in the present paper, is an almost unexplored field. Since the example proposed here - due to its symmetry properties - allows us to obtain a complete determination of the local stability regions and the local 
bifurcation curves in the parameters space by simple analytical expressions, it may constitute a good starting point for a more complete study of the global properties and bifurcations of the complex attractors and their basins.

The paper is organized as follows. In Section 2 a description of the model is provided, and the threedimensional map whose iteration gives the time evolution of the repeated game is defined. In Section 3 the fixed points of the map, which are Nash equilibria of the game, are computed. In Section 4 we describe the symmetry properties of the model with identical players. In Section 5 we study the local stability of the fixed points, together with their local bifurcations. In Section 6 the basins of the coexisting stable equilibria are numerically studied and some changes of their topological properties are evidenced. In Section 7 numerical simulations of the model are given in the case of coexisting quasiperiodic or chaotic attractors. In Section 8 the case of heterogeneous players is considered, and some phenomena related to the loss of symmetry properties are discussed. We end the paper with a discussion of the significance of our results from an economic as well as from a mathematical point of view.

\section{The model}

To introduce our dynamic Cournot oligopoly model with three firms, let us consider a quantitysetting triopoly, where the firms produce a homogeneous good. The environment of our dynamic game is described by the strategy sets of the firms, from which they can choose their output quantities $q_{i}$, $i=1,2,3$, and the resulting instantaneous payoffs $U_{i}\left(q_{1}, q_{2}, q_{3}\right), i=1,2,3$. The functions $r_{i}, i=1,2$, 3 are called 'Cournot reaction functions' or best replies if ${ }^{6}$ :

$$
\begin{aligned}
& \max _{q_{1}} U_{1}\left(q_{1}, q_{2}, q_{3}\right)=U_{1}\left(r_{1}\left(q_{2}, q_{3}\right), q_{2}, q_{3}\right) \\
& \max _{q_{2}} U_{2}\left(q_{1}, q_{2}, q_{3}\right)=U_{2}\left(q_{1}, r_{2}\left(q_{3}, q_{1}\right), q_{3}\right) \\
& \max _{q_{3}} U_{3}\left(q_{1}, q_{2}, q_{3}\right)=U_{3}\left(q_{1}, q_{2}, r_{3}\left(q_{1}, q_{2}\right)\right)
\end{aligned}
$$

These reactions functions specify each firm's optimal output quantity for given quantities of the opponents. In order to introduce dynamics into the model, we first look again at the work of Cournot. He considered a dynamic adjustment process, which describes how firms adjust their output quantities in response to the quantities observed in the previous period. This process nowadays is referred to as Best Response Dynamic (or Cournot tâtonnement), and assumes that each firm sets the current output equal to the best response (i.e. current period payoff maximizing choice) to the last period output of its opponents $^{7}$. Formally, the Best Response Dynamic can be described in terms of the reaction functions as:

$$
\begin{aligned}
& q_{1}(t+1)=r_{1}\left(q_{2}(t), q_{3}(t)\right) \\
& q_{2}(t+1)=r_{2}\left(q_{3}(t), q_{1}(t)\right) \\
& q_{3}(t+1)=r_{3}\left(q_{1}(t), q_{2}(t)\right)
\end{aligned}
$$

\footnotetext{
${ }^{6}$ Extensive references for questions about the existence, uniqueness and continuity of the reaction functions can be found in [10].

${ }^{7}$ To establish a relationship to the introduction, where we mentioned that firms react to the expected quantities of the rivals, we note that the Best Response Dynamic is obtained if we assume that each firm expects that the rivals will offer in the current period the same quantities as in the previous period. In the literature such type of expectations are called 'naive', which can be interpreted to mean that the firm's prediction of the competitors' choice is not very sophisticated.
} 
In what follows, however, we do not consider the Best Response Dynamic. Instead we assume that the firms only partially adjust to the Best Response. This incorporates the fact that firms might be insecure if their forecast of the opponent's choices is correct. Furthermore, our formulation might also capture some element of inertia in the decision making process of the firms. In these cases the competitors may use an adjustment process which is more conservative than the Best Response. Formally, we assume that competitors do not immediately offer the optimal quantity they computed on the basis of the (bounded rationality) profit maximization problem, but that they adjust the previous quantity in the direction of the computed one according to:

$$
\begin{aligned}
& q_{1}(t+1)=q_{1}(t)+\lambda_{1}\left(r_{1}\left(q_{2}(t), q_{3}(t)\right)-q_{1}(t)\right) \\
& q_{2}(t+1)=q_{2}(t)+\lambda_{2}\left(r_{2}\left(q_{3}(t), q_{1}(t)\right)-q_{2}(t)\right) \\
& q_{3}(t+1)=q_{3}(t)+\lambda_{3}\left(r_{3}\left(q_{1}(t), q_{2}(t)\right)-q_{3}(t)\right)
\end{aligned}
$$

The parameters $\lambda_{i} \in[0,1], i=1,2$, represent the speeds of adjustment, a measure of a firm's trust in its own prediction or of the degree of inertia. Note that if $\lambda_{i}=1, i=1,2,3$, (instantaneous adjustment) then we get as a limiting case the usual Cournot tâtonnement Eq. (2). In this particular case, the decision of each firm only depends on the competitors' realized quantities of the previous period. This is the case which has been considered by Puu [20,21].

To obtain a complete characterization of the model, we have to specify the reaction functions $r_{i}$, $i=1,2,3$. As one particular example we consider in this paper the following specification of the reaction functions:

$$
\begin{aligned}
& r_{1}\left(q_{2}, q_{3}\right)=\mu_{1}\left[q_{2}\left(1-q_{2}\right)+q_{3}\left(1-q_{3}\right)\right] \\
& r_{2}\left(q_{3}, q_{1}\right)=\mu_{2}\left[q_{3}\left(1-q_{3}\right)+q_{1}\left(1-q_{1}\right)\right] \\
& r_{3}\left(q_{1}, q_{2}\right)=\mu_{3}\left[q_{1}\left(1-q_{1}\right)+q_{2}\left(1-q_{2}\right)\right]
\end{aligned}
$$

These functional forms of the reaction functions can be derived by assuming a linear demand function and a suitable definition of the cost function of the firms. The cost function of the firms incorporates an interfirm externality as described above, and the parameters $\mu_{i}, i=1,2,3$ represent the extent of this externality. Since the reaction curves can be derived by the same procedure as introduced in [16], we do not reiterate it here, but instead leave the task to the interested reader.

\section{Nash equilibria of the game}

The time evolution of the oligopoly game Eq. (3) with reaction functions Eq. (4) is obtained by the iteration of the three-dimensional map $T:\left(q_{1}, q_{2}, q_{3}\right) \rightarrow\left(q_{1}^{\prime}, q_{2}^{\prime}, q_{3}^{\prime}\right)$ :

$$
T:\left\{\begin{array}{l}
q_{1}^{\prime}=\left(1-\lambda_{1}\right) q_{1}+\lambda_{1} \mu_{1}\left[q_{2}\left(1-q_{2}\right)+q_{3}\left(1-q_{3}\right)\right] \\
q_{2}^{\prime}=\left(1-\lambda_{2}\right) q_{2}+\lambda_{2} \mu_{2}\left[q_{3}\left(1-q_{3}\right)+q_{1}\left(1-q_{1}\right)\right] \\
q_{3}^{\prime}=\left(1-\lambda_{3}\right) q_{3}+\lambda_{3} \mu_{3}\left[q_{1}\left(1-q_{1}\right)+q_{2}\left(1-q_{2}\right)\right]
\end{array}\right.
$$

with $\mu_{i}>0$ and $\lambda_{i} \in[0,1]$, where ' denotes the unit-time advancement operator. That is, if the right hand side includes quantities of period $t$ then the left hand side represents quantities of period $(t+1)$. Starting from a given initial condition

$$
\left(q_{1}(0), q_{2}(0), q_{3}(0)\right)=\left(q_{1_{0}}, q_{2_{0}}, q_{3_{0}}\right)
$$


the iteration of Eq. (5) uniquely determines an infinite sequence of points, the trajectory:

$$
\tau\left(q_{1_{0}}, q_{2_{0}}, q_{3_{0}}\right)=\left\{\left(q_{1}(t), q_{2}(t), q_{3}(t)\right)=T^{t}\left(q_{1_{0}}, q_{2_{0}}, q_{3_{0}}\right), \quad t=0,1,2, \ldots\right\} .
$$

The map Eq. (5) is a three-dimensional non-invertible map which depends on six parameters. Noninvertible means that even if the image of a point $\left(q_{1}, q_{2}, q_{3}\right)$ is uniquely determined as $\left(q_{1}^{\prime}, q_{2}^{\prime}, q_{3}^{\prime}\right)=T\left(q_{1}, q_{2}, q_{3}\right)$, the set of points which are mapped to a given $\left(q_{1}^{\prime}, q_{2}^{\prime}, q_{3}^{\prime}\right)$, i.e. the rank-1 preimages $T^{-1}\left(q_{1}^{\prime}, q_{2}^{\prime}, q_{3}^{\prime}\right)$, may be more than one, i.e. $T^{-1}$ is a multivalued relation. In fact, the rank-1 preimages of $\left(q_{1}^{\prime}, q_{2}^{\prime}, q_{3}^{\prime}\right)$ may be up to eight, since they are obtained by solving the eighth degree algebraic system Eq. (5) with respect to the unknowns $\left(q_{1}, q_{2}, q_{3}\right)$ :

We are concerned with the qualitative changes of the asymptotic (or long-run) dynamics of the iterated map and the influence of the initial conditions as the parameters vary. As usual, the first step to start the qualitative study of the asymptotic behavior of a dynamic model is the localization of the steady states of the dynamical system and the determination of the sets of parameters for which they are locally stable. The fixed points of the map Eq. (5), which are Nash equilibria for the oligopoly, are the solutions of the following algebraic system of degree 8 :

$$
\begin{aligned}
& \mu_{1}\left[q_{2}\left(1-q_{2}\right)+q_{3}\left(1-q_{3}\right)\right]=q_{1} \\
& \mu_{2}\left[q_{1}\left(1-q_{1}\right)+q_{3}\left(1-q_{3}\right)\right]=q_{2} \\
& \mu_{3}\left[q_{2}\left(1-q_{2}\right)+q_{1}\left(1-q_{1}\right)\right]=q_{3}
\end{aligned}
$$

It is worth noticing that the system Eq. (8) does not depend on the rate parameters $\lambda_{i}$. An analytic solution of Eq. (8) is not an easy task. Two real solutions of Eq. (8) exist for each value of $\mu_{i}, i=1,2$, 3: one is given by:

$$
E_{1}=(0,0,0)
$$

and another one must exist, since the degree of the algebraic system Eq. (8) is even.

A complete analytical solution of the system Eq. (8) can be found under the assumption:

$$
\mu_{1}=\mu_{2}=\mu_{3}=\mu
$$

which means that the positive externality which the actions of each firm exerts on the payoffs of the other firms is equal. Under this assumption, the second fixed point which exists for each value of $\mu$ is given by the symmetric ${ }^{8}$ Nash equilibrium:

$$
E_{2}=\left(1-\frac{1}{2 \mu}, 1-\frac{1}{2 \mu}, 1-\frac{1}{2 \mu}\right)
$$

Moreover, other six fixed points exist provided that:

$$
\mu>\sqrt{2}+\frac{1}{2}
$$

In fact, if Eq. (12) is satisfied, the function

$$
\Psi(\mu)=4 \mu^{2}-4 \mu-7
$$

\footnotetext{
${ }^{8}$ Symmetric means that it is characterized by three identical outputs of the competing firms.
} 
is positive, and the following fixed points exist:

$$
\begin{aligned}
& E_{3}=\left(\frac{1+2 \mu+\sqrt{\Psi}}{4 \mu}, \frac{1+2 \mu+\sqrt{\Psi}}{4 \mu}, \frac{3+2 \mu-\sqrt{\Psi}}{4 \mu}\right) \\
& E_{4}=\left(\frac{1+2 \mu-\sqrt{\Psi}}{4 \mu}, \frac{1+2 \mu-\sqrt{\Psi}}{4 \mu}, \frac{3+2 \mu+\sqrt{\Psi}}{4 \mu}\right) \\
& E_{5}=\left(\frac{3+2 \mu-\sqrt{\Psi}}{4 \mu}, \frac{1+2 \mu+\sqrt{\Psi}}{4 \mu}, \frac{1+2 \mu+\sqrt{\Psi}}{4 \mu}\right) \\
& E_{6}=\left(\frac{3+2 \mu+\sqrt{\Psi}}{4 \mu}, \frac{1+2 \mu-\sqrt{\Psi}}{4 \mu}, \frac{1+2 \mu-\sqrt{\Psi}}{4 \mu}\right) \\
& E_{7}=\left(\frac{1+2 \mu+\sqrt{\Psi}}{4 \mu}, \frac{3+2 \mu-\sqrt{\Psi}}{4 \mu}, \frac{1+2 \mu+\sqrt{\Psi}}{4 \mu}\right) \\
& E_{8}=\left(\frac{1+2 \mu-\sqrt{\Psi}}{4 \mu}, \frac{3+2 \mu+\sqrt{\Psi}}{4 \mu}, \frac{1+2 \mu-\sqrt{\Psi}}{4 \mu}\right) .
\end{aligned}
$$

\section{Remark 1.}

At $\mu=1 / 2$ we have $E_{1}=E_{2}=(0,0,0)$;

At $\mu=1 / 2+\sqrt{2}$ we have $E_{3}=E_{4}=((3+\sqrt{2}) / 7,(3+\sqrt{2}) / 7,(2+\sqrt{2}) / 7), E_{5}=E_{6}=((2+$ $\sqrt{2}) / 7,(3+\sqrt{2}) / 7,(3+\sqrt{2}) / 7), E_{7}=E_{8}=((3+\sqrt{2}) / 7,(2+\sqrt{2}) / 7,(3+\sqrt{2}) / 7) ;$

At $\mu=2$ we have $E_{2}=E_{3}=E_{5}=E_{7}=(3 / 4,3 / 4,3 / 4)$.

\section{Homogeneous behavior and symmetries of the dynamical system}

In the following we consider the case of homogeneous behavior:

$$
\lambda_{1}=\lambda_{2}=\lambda_{3}=\lambda
$$

which has been the main subject of analysis in the literature, see [2,5,16] for further references.

Under the assumptions Eqs. (10) and (20) the six-parameter map Eq. (5) reduces to the simpler twoparameter map:

$$
T_{\mathrm{H}}:\left\{\begin{array}{l}
q_{1}^{\prime}=(1-\lambda) q_{1}+\lambda \mu\left[q_{2}\left(1-q_{2}\right)+q_{3}\left(1-q_{3}\right)\right] \\
q_{2}^{\prime}=(1-\lambda) q_{2}+\lambda \mu\left[q_{3}\left(1-q_{3}\right)+q_{1}\left(1-q_{1}\right)\right] \\
q_{3}^{\prime}=(1-\lambda) q_{3}+\lambda \mu\left[q_{1}\left(1-q_{1}\right)+q_{2}\left(1-q_{2}\right)\right]
\end{array}\right.
$$


with $(\mu, \lambda) \in \Omega$, where:

$$
\Omega=\{(\mu, \lambda) \mid \mu>0,0 \leq \lambda \leq 1\}
$$

Although the dynamical system obtained by the iteration of the map Eq. (21) gives the time evolution of a special case of an oligopoly, one which is characterized by three identical competitors, it constitutes a benchmark case which is a useful starting point for the understanding of the dynamical properties of the more complex model represented by Eq. (5).

The map Eq. (21) has a number of symmetry properties, related to the fact that, the three players being identical, the game remains exactly the same if the players are exchanged. We now try to formalize this idea.

In general, a linear transformation $M: \mathbb{R}^{3} \rightarrow \mathbb{R}^{3}$ is called a symmetry of the map $T_{\mathrm{H}}$ if $M$ and $T_{\mathrm{H}}$ commute, that is:

$$
M \circ T_{\mathrm{H}}=T_{\mathrm{H}} \circ M
$$

Due to the identity of the players, an evident group of symmetries of the map Eq. (21) is represented by the group of permutation matrices:

$$
D_{3}=\left\{M_{123}, M_{132}, M_{321}, M_{213}, M_{312}, M_{231}\right\}
$$

where $M_{i j k}$ are $3 \times 3$ matrices such that:

$$
M_{i j k}\left[\begin{array}{l}
q_{1} \\
q_{2} \\
q_{3}
\end{array}\right]=\left[\begin{array}{l}
q_{i} \\
q_{j} \\
q_{k}
\end{array}\right] .
$$

Apart from the trivial symmetry $M_{123}=I$, which is the identity transformation, there are five interesting symmetries. The first three correspond to permutations of two of the three coordinates, respectively, given by:

$$
P_{1}\left(q_{1}, q_{2}, q_{3}\right)=\left(q_{1}, q_{3}, q_{2}\right), \quad P_{2}\left(q_{1}, q_{2}, q_{3}\right)=\left(q_{3}, q_{2}, q_{1}\right), \quad P_{3}\left(q_{1}, q_{2}, q_{3}\right)=\left(q_{2}, q_{1}, q_{3}\right)
$$

which represent reflections through the planes:

$$
\Pi_{1}=\left\{\left(q_{1}, q_{2}, q_{3}\right) \mid q_{2}=q_{3}\right\}, \quad \Pi_{2}=\left\{\left(q_{1}, q_{2}, q_{3}\right) \mid q_{1}=q_{3}\right\}, \quad \Pi_{3}=\left\{\left(q_{1}, q_{2}, q_{3}\right) \mid q_{1}=q_{2}\right\},
$$

respectively.

The fact that the map Eq. (21) commutes with $P_{i}$, i.e. $P_{i}\left(T_{\mathrm{H}}\left(q_{1}, q_{2}, q_{3}\right)\right)=T_{\mathrm{H}}\left(P_{i}\left(q_{1}, q_{2}, q_{3}\right)\right)$, implies that any orbit $G=\left\{\left(q_{1 n}, q_{2 n}, q_{3 n}\right)\right\}$ of $T_{\mathrm{H}}$ is either symmetric with respect to the plane $\Pi_{i}$, i.e. for each point $\left(q_{1 n}, q_{2 n}, q_{3 n}\right) \in G$ also $P_{i}\left(q_{1 n}, q_{2 n}, q_{3 n}\right) \in G$, or the set $P_{i}(G)=\left\{P_{i}\left(q_{1 n}, q_{2 n}, q_{3 n}\right)\right\}$, symmetric of $G$ with respect to the plane $\Pi_{i}$, is an orbit of $T$ as well.

Furthermore, a dynamical system with a symmetry $M$ has a linear invariant subspace, given by the set of fixed points of $M$ (see e.g. [8]). In our case, as $T_{\mathrm{H}}$ commutes with $P_{i}, i=1,2,3$, each plane $\Pi_{i}$ is an invariant submanifold for the map $T_{\mathrm{H}}$, i.e. $T_{\mathrm{H}}\left(\Pi_{i}\right) \subseteq \Pi_{i}$. The trajectories embedded inside $\Pi_{i}$ are governed by the restriction of $T$ to $\Pi_{i}$, given by a two-dimensional map. For example, let us consider the invariant plane $\Pi_{1}$. Any trajectory starting with an initial condition belonging to $\Pi_{1}$, i.e. $q_{2_{0}}=q_{3_{0}}$, is characterized by $q_{2}(t)=q_{3}(t)$ for each $t \geq 0$, and is governed by the restriction of $T_{\mathrm{H}}$ to $\Pi_{1}$, which can 
be identified with the two-dimensional map $T_{1}:\left(q_{1}, q\right) \rightarrow\left(q_{1}^{\prime}, q^{\prime}\right)$ given by:

$$
T_{1}:\left\{\begin{array}{l}
q_{1}^{\prime}=(1-\lambda) q_{1}+2 \lambda \mu q(1-q) \\
q^{\prime}=(1-\lambda) q+\lambda \mu\left[q_{1}\left(1-q_{1}\right)+q(1-q)\right]
\end{array}\right.
$$

obtained by setting $q_{2}=q_{3}=q$ and $q_{2}^{\prime}=q_{3}^{\prime}=q^{\prime}$ in Eq. (21). Analogous arguments apply to the other invariant planes and the respective restrictions.

The trajectories embedded into an invariant plane $\Pi_{i}$ will be called partially synchronized, since the time evolutions described by such trajectories are characterized by identical choices of two competitors at each time period, that is, two of the three players move in a synchronized way. Of course, the intersection of the three invariant planes $\Pi_{i}, i=1,2,3$, given by the diagonal:

$$
\Delta=\left\{(q, q, q) \in \mathbb{R}^{3}\right\},
$$

or line of equal outputs, is an invariant one-dimensional submanifold for $T_{\mathrm{H}}$, i.e. $T_{\mathrm{H}}(\Delta) \subseteq \Delta$. This property expresses the trivial statement that if the three identical producers start with equal productions $q_{1}(0)=q_{2}(0)=q_{3}(0)$ then they will behave identically for each future time, i.e. $q_{1}(t)=q_{2}(t)=q_{3}(t)$ for each $t \geq 0$. The trajectories embedded inside $\Delta$ will be called fully synchronized trajectories because they are characterized by identical production choices of all the competitors at each time period. The synchronized trajectories are governed by the restriction of $T_{H}$ to $\Delta,\left.T_{H}\right|_{\Delta}: \Delta \rightarrow \Delta$, which can be identified with the one-dimensional map:

$$
q^{\prime}=g(q)=(1-\lambda+2 \mu \lambda) q-2 \lambda \mu q^{2}
$$

obtained by setting $q_{1}=q_{2}=q_{3}=q$ and $q_{1}^{\prime}=q_{2}^{\prime}=q_{3}^{\prime}=q^{\prime}$ in Eq. (5). The map Eq. (25), whose dynamics summarize the common behavior of the three identical firms, is conjugated to the standard logistic map:

$$
z^{\prime}=a z(1-z)
$$

through the linear transformation:

$$
z=\frac{2 \lambda \mu}{1-\lambda+2 \lambda \mu} q
$$

and the parameters of the map Eq. (25) are related to the parameter $a$ of the standard logistic by the relation:

$$
a=1-\lambda+2 \lambda \mu .
$$

The knowledge of the dynamical properties of the restrictions to the invariant lower-dimensional submanifolds gives important suggestions about the dynamic behavior of the three-dimensional map Eq. (21). In fact, the properties of the one-dimensional restriction to the invariant line $\Delta$ are wellknown, being conjugated to the standard logistic map, and the properties of the two-dimensional restrictions to the invariant planes $\Pi_{i}$ can be obtained on the basis of the local and global methods for the study of non-invertible maps of the plane, which are now sufficiently known (see e.g. [1,17] for many examples, see also [5,11] for maps which are very similar to Eq. (23)). 
The last two permutation matrices of $D_{3}$ correspond to cyclic permutations of the three coordinates:

$$
R_{3}^{1}:\left(q_{1}, q_{2}, q_{3}\right) \rightarrow\left(q_{3}, q_{1}, q_{2}\right) \text { and } R_{3}^{2}:\left(q_{1}, q_{2}, q_{3}\right) \rightarrow\left(q_{2}, q_{3}, q_{1}\right)
$$

and represent rotations of $(2 / 3) \pi$ around $\Delta$. The fact that the map $T_{\mathrm{H}}$ also commutes with $R_{3}^{i}, i=1,2$, implies that for any orbit of Eq. (21) which is not embedded into $\Delta$ two other orbits must exist, which are rotated by $(2 / 3) \pi$ with respect to $\Delta$.

Remark 2. From the arguments given above it trivially follows that in the case of homogeneous behavior Eq. (20) all the fixed points of the symmetric map Eq. (21), i.e. Nash equilibria of the Cournot game, belong to invariant submanifolds. In fact, $E_{1}$ and $E_{2}$ belong to $\Delta, E_{3}$ and $E_{4}$ belong to $\Pi_{3}, E_{5}$ and $E_{6}$ belong to $\Pi_{1}, E_{7}$ and $E_{8}$ belong to $\Pi_{2}$.

\section{Local stability and local bifurcations of the Nash equilibria}

In this section we consider the repeated Cournot game with three identical oligopolists, whose time evolution is obtained by the iteration of the map Eq. (21). We study the local stability of the Nash equilibria $E_{i}, i=1, \ldots, 8$, which are the fixed points of the map Eq. (21), as the two parameters $\mu$ and $\lambda$ vary in the parameters space $\Omega$ defined in Eq. (22). The study of the local stability of a fixed point $E_{i}$ is based on the localization, on the complex plane, of the eigenvalues of the Jacobian matrix:

$$
D T_{\mathrm{H}}\left(q_{1}, q_{2}, q_{2}\right)=\left[\begin{array}{llc}
1-\lambda & \mu \lambda\left(1-2 q_{2}\right) & \mu \lambda\left(1-2 q_{3}\right) \\
\mu \lambda\left(1-2 q_{1}\right) & 1-\lambda & \mu \lambda\left(1-2 q_{3}\right) \\
\mu \lambda\left(1-2 q_{1}\right) & \mu \lambda\left(1-2 q_{2}\right) & 1-\lambda
\end{array}\right]
$$

computed at $E_{i}$. If the three eigenvalues $z_{k}, k=1,2,3$, of $D T_{\mathrm{H}}\left(E_{i} ; \mu, \lambda\right)$ are all inside the unit circle of the complex plane, i.e. $\left|z_{k}\right|<1, k=1,2,3$, then $E_{i}$ is locally asymptotically stable.

The Jacobian matrix Eq. (29) becomes a symmetric matrix when computed at the fixed points $E_{1}$ and $E_{2}$ belonging to the line $\Delta$ of equal quantities. This implies that at $E_{1}$ and $E_{2}$ the eigenvalues are always real. At $E_{1}$ the characteristic equation becomes:

$$
z^{3}-3(1-\lambda) z^{2}+3(1-\lambda-\mu \lambda)(1-\lambda+\mu \lambda) z-(1-\lambda-\mu \lambda)^{2}(1-\lambda+2 \mu \lambda)=0
$$

and the eigenvalues are:

$$
z_{1}\left(E_{1}\right)=1-\lambda+2 \mu \lambda, \quad z_{2}\left(E_{1}\right)=z_{3}\left(E_{1}\right)=1-\lambda-\mu \lambda
$$

We have:

- $z_{1}\left(E_{1}\right) \geq 0 \forall(\mu, \lambda) \in \Omega$ and $z_{1}\left(E_{1}\right)<1$ iff $\mu<1 / 2$;

- $z_{2}\left(E_{1}\right)<1 \forall(\mu, \lambda) \in \Omega$ and $z_{2}\left(E_{1}\right)>-1$ iff $\lambda(\mu+1)<2$.

Hence, the stability domain of $E_{1}$ in parameter space is given by:

$$
\Omega^{\mathrm{s}}\left(E_{1}\right)=\left\{(\mu, \lambda) \in \Omega \mid \mu<\frac{1}{2}\right\} .
$$

Notice that the eigenvalue $z_{1}\left(E_{1}\right)$ coincides with the multiplier at $q=0$ of the restriction Eq. (25) of $T_{\mathrm{H}}$ to the invariant line $\Delta$. 
At $E_{2}$ the characteristic equation becomes:

$$
z^{3}-3(1-\lambda) z^{2}+3(1-2 \lambda+\mu \lambda)(1-\mu \lambda) z-(1+\lambda-2 \mu \lambda)(1-2 \lambda+\mu \lambda)^{2}=0
$$

and the eigenvalues are:

$$
z_{1}\left(E_{2}\right)=1+\lambda-2 \mu \lambda, \quad z_{2}\left(E_{2}\right)=z_{3}\left(E_{2}\right)=1-2 \lambda+\mu \lambda
$$

Again, the eigenvalue $z_{1}\left(E_{2}\right)$ coincides with the multiplier at $q=(1-1 /(2 \mu))$ of the restriction Eq. (25) of $T_{\mathrm{H}}$ to the invariant line $\Delta$. We have:

- $z_{1}\left(E_{2}\right)<1$ iff $\mu>1 / 2$;

- $z_{1}\left(E_{2}\right)>-1$ iff $\lambda(2 \mu-1)<2, z_{2}\left(E_{1}\right)>-1 \forall(\mu, \lambda) \in \Omega$ and $z_{2}\left(E_{1}\right)<1$ iff $\mu<2$.

Hence, the stability domain of $E_{2}$ in parameter space is given by:

$$
\Omega^{\mathrm{s}}\left(E_{2}\right)=\left\{(\mu, \lambda) \in \Omega \mid \frac{1}{2}<\mu<2 \text { and } \lambda(2 \mu-1)<2\right\} .
$$

At $\mu=1 / 2$ we have $E_{1}=E_{2}$, and a transcritical bifurcation occurs: as $\mu$ increases through the bifurcation value $1 / 2, E_{1}$ is changed from a stable node into a saddle point, and $E_{2}$ becomes a stable node.

Also the bifurcation occurring at $\mu=2$ is a transcritical bifurcation, whose nature will be investigated below. Along the curve $\lambda(2 \mu-1)=2$ we have $z_{1}\left(E_{2}\right)=-1$. If the parameters vary so that this curve is crossed with increasing values of $\mu$ or $\lambda$ then $E_{2}$ loses stability through a flip (or period doubling) bifurcation.

In order to study the stability properties of the fixed points $E_{i}, i=3, \ldots, 8$, we subdivide them into two groups: $U=\left\{E_{3}, E_{5}, E_{7}\right\}$ and $S=\left\{E_{4}, E_{6}, E_{8}\right\}$. Each group is characterized by the same stability properties. In fact, the Jacobian matrix computed at each of the fixed points $E_{i} \in U$ is the same, given by:

$$
D T_{\mathrm{H}}\left(E_{i} ; \mu, \lambda\right)=\left[\begin{array}{llc}
1-\lambda & -(\lambda / 2)(1+\sqrt{\Psi}) & (\lambda / 2)(\sqrt{\Psi}-3) \\
-(\lambda / 2)(1+\sqrt{\Psi}) & 1-\lambda & (\lambda / 2)(\sqrt{\Psi}-3) \\
-(\lambda / 2)(1+\sqrt{\Psi}) & -(\lambda / 2)(1+\sqrt{\Psi}) & 1-\lambda
\end{array}\right], \quad i=3,5,7
$$

where $\Psi=\Psi(\mu)$ is the function defined in Eq. (13), and also the Jacobian matrix computed at each of the fixed points $E_{i} \in S$ is the same, given by:

$$
D T_{\mathrm{H}}\left(E_{i} ; \mu, \lambda\right)=\left[\begin{array}{llc}
1-\lambda & (\lambda / 2)(\sqrt{\Psi}-1) & -(\lambda / 2)(\sqrt{\Psi}+3) \\
(\lambda / 2)(\sqrt{\Psi}-1) & 1-\lambda & -(\lambda / 2)(\sqrt{\Psi}+3) \\
(\lambda / 2)(\sqrt{\Psi}-1) & (\lambda / 2)(\sqrt{\Psi}-1) & 1-\lambda
\end{array}\right] \quad i=4,6,8
$$

We now study the conditions for the local stability and local bifurcations of $E_{3}$, which, according to the arguments given above, also hold for the fixed points $E_{5}$ and $E_{7}$. The eigenvalues of $D T_{\mathrm{H}}\left(E_{3} ; \mu, \lambda\right)$ 
are:

$$
\begin{aligned}
& z_{1}\left(E_{3}\right)=\frac{1}{2}(2-\lambda+\lambda \sqrt{\Psi(\mu)}) \\
& z_{2}\left(E_{3}\right)=\frac{1}{4}(4-\lambda \sqrt{\Psi(\mu)}-5 \lambda-\lambda \sqrt{(25-7 \sqrt{\Psi(\mu)}(1+\sqrt{\Psi(\mu)})}) \\
& z_{3}\left(E_{3}\right)=\frac{1}{4}(4-\lambda \sqrt{\Psi(\mu)}-5 \lambda+\lambda \sqrt{(25-7 \sqrt{\Psi(\mu)}(1+\sqrt{\Psi(\mu)})})
\end{aligned}
$$

and it is easy to realize that the fixed points $E_{i} \in U$ are always unstable.

In fact, at least one eigenvalue with modulus greater than one exists for each $\mu>1+\sqrt{2}$, being $z_{3}\left(E_{3}\right)>1$ for $\mu_{1}<\mu<2$, where $\mu_{1}=1 / 2+\sqrt{2}$, and $z_{1}\left(E_{3}\right)>1$ for $\mu>2$.

Notice that at $\mu=1+\sqrt{2}$ we have $z_{3}\left(E_{3}\right)=1$ and $z_{1}\left(E_{3}\right)=z_{2}\left(E_{3}\right)=1-5 / 2 \lambda$.

At $\mu=2$ we have $z_{1}=z_{3}=1$ and $z_{2}=1-3 \lambda$. As remarked at the end of Section 3 , at $\mu=2$ we have $E_{3}=E_{5}=E_{7}=E_{2}$, so the bifurcation occurring at $\mu=2$ is a transcritical bifurcation at which $E_{2}$ is transformed, for increasing values of $\mu$, from a stable node into a saddle point, due to the exit of $z_{2}\left(E_{2}\right)$ from the unit circle through the value $z_{2}\left(E_{2}\right)=1$ and the simultaneous entrance, through the same value, of $z_{3}\left(E_{3}\right)$.

We also notice that $z_{2}\left(E_{3}\right)$ and $z_{3}\left(E_{3}\right)$ are real for $\sqrt{\Psi(\mu)} \leq 25 / 7$, i.e. for $\mu_{1} \leq \mu \leq 1 / 2+3 \sqrt{113} / 14$ and they are complex conjugated for $\mu>1 / 2+3 \sqrt{113} / 14$.

We now study the conditions for the local stability and local bifurcations of $E_{4}$, which, according to the arguments given above, also hold for the fixed points $E_{6}$ and $E_{8}$. The eigenvalues of $D T_{\mathrm{H}}\left(E_{4} ; \mu, \lambda\right)$ are:

$$
\begin{aligned}
& z_{1}\left(E_{4}\right)=\frac{1}{2}(2-\lambda-\lambda \sqrt{\Psi(\mu)}) \\
& z_{2}\left(E_{4}\right)=\frac{1}{4}(4-\lambda \sqrt{\Psi(\mu)}-5 \lambda-\lambda \sqrt{(25+7 \sqrt{\Psi(\mu)}(1-\sqrt{\Psi(\mu)})}) \\
& z_{3}\left(E_{4}\right)=\frac{1}{4}(4+\lambda \sqrt{\Psi(\mu)}-5 \lambda+\lambda \sqrt{(25+7 \sqrt{\Psi(\mu)}(1-\sqrt{\Psi(\mu)})})
\end{aligned}
$$

The conditions $\left|z_{i}\left(E_{4}\right)\right|<1, i=1,2,3$, define the region of stability $\Omega^{\mathrm{s}}\left(E_{4}\right)=\Omega_{1}^{\mathrm{s}}\left(E_{4}\right) \cup \Omega_{2}^{\mathrm{s}}\left(E_{4}\right)$, where:

$$
\Omega_{1}^{\mathrm{s}}\left(E_{4}\right)=\left\{(\mu, \lambda) \in \Omega \mid \frac{1}{2}+\sqrt{2}<\mu<2 \text { and } 0<\lambda<\lambda_{f}(\mu)\right\}
$$

with:

$$
\lambda_{f}(\mu)=\frac{8}{5-\sqrt{\Psi(\mu)}+\sqrt{(25+7 \sqrt{\Psi(\mu)})(1-\sqrt{\Psi(\mu)})}}
$$

and

$$
\Omega_{2}^{\mathrm{s}}\left(E_{4}\right)=\left\{(\mu, \lambda) \in \Omega \mid \mu>2 \quad \text { and } \quad 0<\lambda<\lambda_{h}(\mu)\right\}
$$




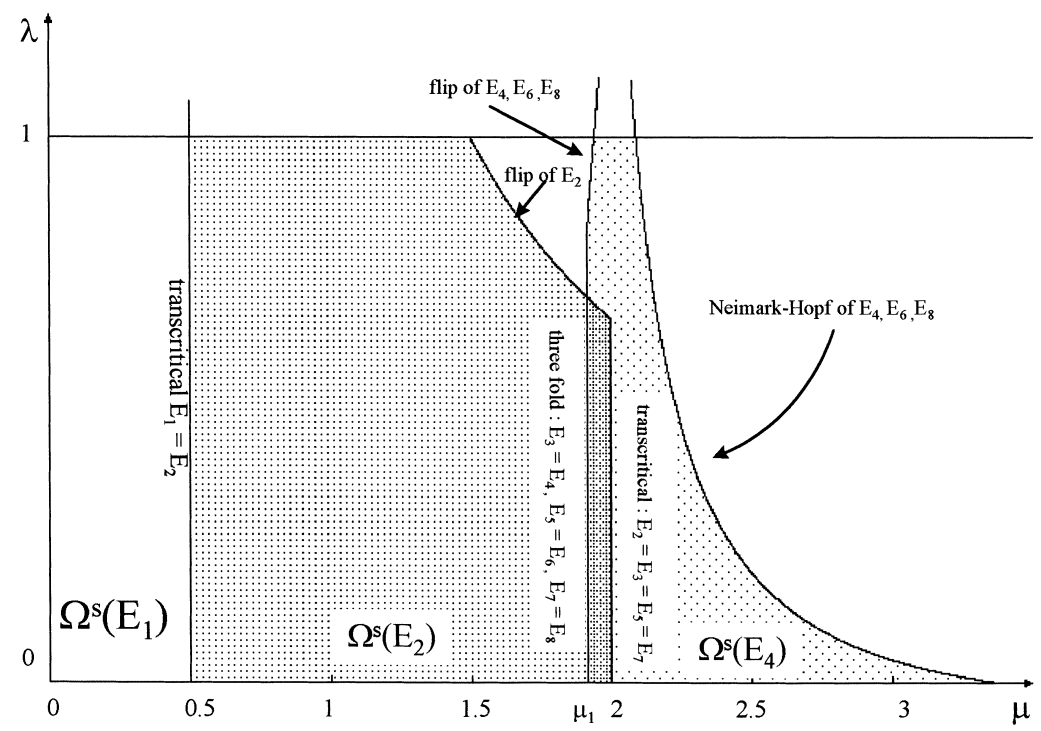

Fig. 1. Space of the parameters $\{$ FUNC $\{\Omega\}\}=\{\mu, \lambda) \mid \mu>0,0 \leq \lambda \leq 1\}$ for the map Eq. (21). $\Omega^{\mathrm{s}}\left(E_{1}\right)$ represent the region where the fixed point $E_{1}$ is asymptotically stable, $\Omega^{\mathrm{s}}\left(E_{2}\right)$ represent the region where the fixed point $E_{2}$ is asymptotically stable, $\Omega^{\mathrm{s}}\left(E_{4}\right)$ represents the common stability region of $E_{4}, E_{6}$ and $E_{8}$.

with

$$
\lambda_{h}(\mu)=\frac{5-\sqrt{\Psi(\mu)}}{\sqrt{\Psi(\mu)}(\sqrt{\Psi(\mu)}+1)}
$$

At $\mu=\mu_{1}=1 / 2+\sqrt{2}$ the fixed point $E_{4}$ (as well as $E_{6}$ and $E_{8}$ ) is created with $z_{3}\left(E_{4}\right)=1$ and it is stable after a slight increase of $\mu$. As remarked above, at the same $\mu$ value also $E_{3}$ (as well as $E_{5}$ and $E_{7}$ ) is created with $z_{3}\left(E_{3}\right)=1$, and $E_{3}=E_{4}$ (as well as $E_{5}=E_{6}$ and $E_{7}=E_{8}$ ). However, $E_{3}, E_{5}$ and $E_{7}$ are unstable (saddle point) after a slight increase of $\mu$, being $z_{3}\left(E_{3}\right)>1$ for $\mu_{1}<\mu<2$. Hence at $\mu=\mu_{1}$ three simultaneous fold (or saddle-node) bifurcations occur at which three pairs of fixed points are created, each pair being constituted by a node and a saddle point.

Along the curve $\lambda=\lambda_{f}(\mu)$ we have $z_{3}\left(E_{4}\right)=-1$, whereas along the curve $\lambda=\lambda h(\mu)$ the eigenvalues $z_{2}\left(E_{4}\right)$ and $z_{3}\left(E_{4}\right)$ are complex conjugated with $\left|z_{2}\left(E_{4}\right)\right|=\left|z_{3}\left(E_{4}\right)\right|=1$. These curves correspond to flip (or period doubling) and Neimark-Hopf bifurcations of $E_{4}$, respectively. Notice that $\lambda_{f}\left(\mu_{1}\right)=4 / 5<1$, and $\lambda_{f}(2)=2>1$, hence a value $\bar{\mu} \in\left(\mu_{1}, 2\right)$ of the parameter $\mu$ exists such that $E_{4}$ is a stable node for each $\lambda \in[0,1]$ provided that $\mu \in(\bar{\mu}, 2)$ (see Fig. 1 ).

We also notice that $z_{2}\left(E_{4}\right)$ and $z_{3}\left(E_{4}\right)$ are real for $\sqrt{\Psi(\mu)} \leq 1$, i.e. for $\mu_{1} \leq \mu \leq 2$, and they are complex conjugated for $\mu>2$. Moreover, we have $\lambda_{h}(2)=2>1$, and $\lambda_{f}(3)=(5-\sqrt{17}) /$ $(17+\sqrt{17})<1$. Hence, a value $\tilde{\mu} \in(2,3)$ of the parameter $\mu$ exists such that $E_{4}$ is a stable focus for each $\lambda \in[0,1]$ provided that $\mu \in(2, \bar{\mu})$ (see Fig. 1 ).

The results on local stability and local bifurcations given above, are summarized by the following statements (see also Fig. 1). 
Proposition 1 (local stability). Let Eqs. (10) and (20) hold. Then

1. the fixed point $E_{1}$ exists for each $(\mu, \lambda) \in \Omega$ and it is a stable node for $(\mu, \lambda) \in \Omega^{\mathrm{s}}\left(E_{1}\right)$, with $\Omega^{\mathrm{s}}\left(E_{1}\right)=$ $\{(\mu, \lambda) \in \Omega \mid \mu(1 / 2)\}$

2. the fixed point $E_{2}$ exists for each $(\mu, \lambda) \in \Omega$ and it is a stable node for $(\mu, \lambda) \in \Omega^{\mathrm{s}}\left(E_{2}\right)$, with $\Omega^{s}\left(E_{2}\right)=\{(\mu, \lambda) \in \Omega \mid(1 / 2)<\mu<2$ and $\lambda(2 \mu-1)<2\}$

3. the fixed points $E_{3}, E_{5}$ and $E_{7}$ exist for $\mu \geq \mu_{1}$, with $\mu_{1}=1 / 2+\sqrt{2}$, and are always unstable;

4. the fixed points $E_{4}, E_{6}$ and $E_{8}$ exist for $\mu \geq \mu_{1}$ and are stable for $(\mu, \lambda) \in \Omega^{\mathrm{s}}\left(E_{4}\right)=\Omega_{1}^{\mathrm{s}}\left(E_{4}\right) \cup$ $\Omega_{2}^{\mathrm{s}}\left(E_{4}\right)$, with:

$$
\Omega_{1}^{\mathrm{s}}\left(E_{4}\right)=\left\{(\mu, \lambda) \in \Omega \mid \mu_{1}<\mu<2 \text { and } 0<\lambda<\lambda_{f}(\mu)\right\}
$$

and

$$
\Omega_{2}^{\mathrm{s}}\left(E_{4}\right)=\left\{(\mu, \lambda) \in \Omega \mid \mu>2 \text { and } 0<\lambda<\lambda_{h}(\mu)\right\}
$$

where $\lambda_{f}(\mu)$ and $\lambda_{h}(\mu)$ are given by Eqs. (35) and (37), respectively. In the region $\Omega_{1}^{\mathrm{s}}\left(E_{4}\right), E_{4}, E_{6}$ and $E_{8}$ are stable nodes, in the region $\Omega_{2}^{\mathrm{s}}\left(E_{4}\right)$ they are stable foci.

Proposition 2 (local bifurcations). Let Eqs. (10) and (20) hold. Then

1. at $\mu=1 / 2, E_{1}=E_{2}$ and a transcritical bifurcation occurs at which, for increasing values of $\mu, E_{1}$ is transformed from a stable node into a saddle point, and $E_{2}$ is transformed from a saddle point into a stable node;

2. at $\mu=1 / 2+\sqrt{2}$, three simultaneous fold bifurcations occur at which, for increasing values of $\mu$, the three pairs of fixed points $\left(E_{3}, E_{4}\right),\left(E_{5}, E_{6}\right)$ and $\left(E_{7}, E_{6}\right)$ are created, which are coincident at the bifurcation value and, for increasing values of $\mu$, they separate with $E_{i}, i=3,5,7$ unstable and $E_{i}, i=4,6,8$ stable;

3. along the curve $\lambda(2 \mu-1)=2$ the fixed point $E_{2}$ undergoes a supercritical flip bifurcation at which, for increasing values of $\mu$ or $\lambda$ it loses stability and a stable cycle of period 2 is created;

4. along the curve $\lambda=\lambda_{f}(\mu)$, where $\lambda_{f}(\mu)$ is given by Eq. (35) with $1 / 2+\sqrt{2}<\mu<2$, the fixed points $E_{i}, i=4,6,8$ undergo a supercritical flip bifurcation at which, for increasing values of $\mu$ or $\lambda$, they lose stability and three stable cycles of period 2 are created;

5. along the curve $\lambda=\lambda_{h}(\mu)$, where $\lambda_{f}(\mu)$ is given by Eq. (37) with $\mu>2$, the fixed points $E_{i}$, $i=4,6,8$ undergo a supercritical Neimark-Hopf bifurcation at which, for increasing values of $\mu$ or $\lambda$, they lose stability and three stable invariant closed curves are created along which the dynamics are periodic or quasi-periodic.

The proofs of these two propositions immediately follow from the arguments given above, except for the supercritical nature of the flip and the Neimark-Hopf bifurcations. We recall that a flip bifurcation is supercritical if a stable 2-cycle is created around the unstable fixed point, subcritical if an unstable 2cycle exists around the stable fixed point and merges with the fixed point at the bifurcation value (see e.g. [12]).

Analogously, a Neimark-Hopf bifurcation is supercritical if an attracting closed invariant curve is created around the unstable fixed point, subcritical if a repelling closed invariant curve exists around the stable fixed point and merges with it at the bifurcation value (see e.g. [12]). The rigorous proof that the flip bifurcation is supercritical requires a center manifold reduction and the evaluation of higher 
order derivatives (up to the third order). Since this is a rather tedious task, we claim numerical evidence for the existence of the stable cycle. Analogously, the rigorous proof of the occurrence of a subcritical Hopf bifurcation requires the evaluation of some long expressions involving derivatives of the map up to order three. Also in this case we claim numerical evidence for the existence of an attracting invariant closed curve just after the Neimark-Hopf bifurcation.

From Proposition 1 it follows that a wide range of parameters exists which gives coexistence of stable Nash equilibria. Since the stability regions $\Omega^{\mathrm{s}}\left(E_{2}\right)$ and $\Omega^{\mathrm{s}}\left(E_{4}\right)$ overlap for $\mu_{1}<\mu<2$ and $\lambda(2 \mu-1)<2$ (the darker region of Fig. 1) in such a region we have four coexisting Nash equilibria, $E_{2}, E_{4}, E_{6}$ and $E_{8}$, which are stable nodes. In the portion $\Omega_{2}^{\mathrm{s}}\left(E_{4}\right)$ of the region $\Omega^{\mathrm{s}}\left(E_{4}\right)$ we have the three coexisting stable Nash equilibria $E_{4}, E_{6}$ and $E_{8}$, which are stable foci. In the portion $\Omega_{1}^{\mathrm{s}}\left(E_{4}\right)$ of $\Omega^{\mathrm{s}}\left(E_{4}\right)$ with $\mu_{1}<\mu<2$ and $\lambda(2 \mu-1)>2$ the three stable Nash equilibria $E_{4}, E_{6}$ and $E_{8}$ exist, which are stable nodes. Accordingly, the questions raised in the introduction arise, namely, to which Nash equilibrium does the evolutionary process described by the market dynamics lead and which role do the initially chosen quantities of the competitors play in this process? As remarked at the end of Section 4, all the fixed points belong to the invariant subsets $\Pi_{i}$. In particular, $E_{2} \in \Delta, E_{4} \in \Pi_{3}, E_{6} \in \Pi_{1}, E_{8} \in \Pi_{2}$. It is also important to remark that at $E_{4}$ we have $q_{3}>q_{1}=q_{2}$, at $E_{6}$ we have $q_{1}>q_{2}=q_{3}$, at $E_{8}$ we have $q_{2}>q_{1}=q_{3}$. Hence, convergence to $E_{4}$ means convergence to an equilibrium outcome at which firm 3 dominates the other two firms. This can be interpreted as a situation where firm 3 becomes the market leader. In the same vein, convergence to $E_{6}$ means convergence to an equilibrium at which the firm 1 dominates the other two competitors, which then take the role of followers. Similar arguments apply to the Nash equilibrium $E_{8}$.

From Proposition 2 we can deduce that just after the flip bifurcation of point (4), as well as after the Neimark-Hopf bifurcation of point (5), we may also have coexistence of attracting sets which are more complex than Nash equilibria, such as periodic or quasi-periodic attractors. Furthermore, numerical simulations show that after these bifurcations, if the parameter values are changed so that they move away from the local bifurcation curves then more and more complex coexisting attractors appear. Of course, such attractors must be such that the symmetry properties described in Section 4 are satisfied, i.e. any attractor must be either symmetric with respect to the planes $\Pi_{i}$ (in particular they may belong to $\Pi_{i}$ ) or other attractors must exist, located in symmetric positions with respect to them. For example, since the three attracting invariant closed curves which are created via the Neimark-Hopf bifurcation belong to the invariant planes $\Pi_{i}$ at their creation, they will remain embedded into the respective invariant planes, to which the three bifurcating fixed points $E_{i}, i=4,6,8$ belong, as far as they exist. The same holds for the more complex attractors which replace the invariant closed curves after their destruction.

\section{Numerical explorations of the basins of the Nash equilibria}

The stability analysis given in Section 5 concerns the attractors of bounded trajectories. However, an attracting set at infinite distance always exists, i.e. diverging trajectories can always be obtained starting from sufficiently high values of $\left\|\left(q_{1}, q_{2}, q_{3}\right)\right\|$.

The coexistence of attracting sets, both at finite and infinite distance, stresses the importance of results on the problem concerning the relationship of the initial conditions Eq. (6) and the long run evolution of the oligopoly game. An answer to this question requires the knowledge of the basins of attraction of the coexisting attractors. 
Let $A$ be an attractor of a map $T:\left(q_{1}, q_{2}, q_{3}\right) \rightarrow\left(q_{1}^{\prime}, q_{2}^{\prime}, q_{3}^{\prime}\right)$, such as a stable fixed point or a stable cycle or a more complex attracting set. The basin of attraction of $A$ is the open set of points which generate trajectories converging to $A$ :

$$
\mathscr{B}(A)=\left\{\left(q_{1}, q_{2}, q_{3}\right) \mid T^{t}\left(q_{1}, q_{2}, q_{3}\right) \rightarrow A \text { as } t \rightarrow+\infty\right\} .
$$

We call basin of infinity $\mathscr{B}(\infty)$ the open set of points that generate diverging trajectories:

$$
\mathscr{B}(\infty)=\left\{\left(q_{1}, q_{2}, q_{3}\right) \mid\left\|T^{t}\left(q_{1}, q_{2}, q_{3}\right)\right\| \rightarrow \infty \text { as } t \rightarrow+\infty\right\} .
$$

The delimitation of the basins of attraction requires a study of the global dynamical properties of the dynamical system, i.e. a study which is not based on the linear approximation of the map. In particular, if the map is non-invertible, the existence of several distinct rank-1 preimages of a given point implies that the basins may be sets with complicated topological structures, like non-connected or multiplyconnected sets (see $[1,17,18]$ ). This can be intuitively understood on the basis of the following arguments. Let $A$ be an attractor for an iterated map $T$. This means that a neighborhood $U(A)$ exists whose points converge to $A$. Of course $U(A) \subseteq \mathscr{B}(A)$, but also the points of the phase space which are mapped inside $U$ after a finite number $n$ of iterations of $T$ belong, for any $n$, to $\mathscr{B}(A)$. So, the total basin of $A$ (or more briefly the basin of $A$ ) is given by:

$$
\mathscr{B}(A)=\bigcup_{n=0}^{\infty} T^{-n}(U(A))
$$

where $T^{-1}(x)$ represent the set of the rank-1 preimages of $x$ (i.e. the points mapped to $x$ by $T$ ), and $T^{-n}(x)$ represent the set of the rank- $n$ preimages of $x$ (i.e. the points mapped to $x$ after $n$ repeated applications of $T$ ). If $T$ is a non-invertible map, the total basin may be non-connected because $U(A)$, or its preimages, may have several rank-1 preimages, obtained by the action of distinct inverses, some of which may be disjoint from $U(A)$. The greatest connected portion of $\mathscr{B}(A)$ which includes $A$ is called the immediate basin of $A$.

The presence of non-connected basins is an important property in applications because it reveals a rather counterintuitive situation, due to the presence of sets of points which generate trajectories converging to a given attractor, surrounded by points belonging to the basin of another attractor. For example, in our triopoly model it may happen that firms eventually use equilibrium strategies $\left(\bar{q}_{1}, \bar{q}_{2}\right.$, $\bar{q}_{3}$ ), although the initially chosen actions were 'far away' from this Nash equilibrium. On the other hand, if the initially chosen quantities are 'close' to this Nash equilibrium, then firms eventually choose some different equilibrium strategies $\left(\tilde{q}_{1}, \tilde{q}_{2}, \tilde{q}_{3}\right)$. Hence, it is important to detect, by analytical and/or numerical methods, the occurrence of global bifurcations at which a basin is transformed from a simply connected into a non-connected set. For two-dimensional maps, some general results for the study of such bifurcations, based on the method of critical curves, a powerful tool for the study of the global properties of non-invertible maps of the plane, are given in $[1,17,18]$. Some applications of these results to a Cournot duopoly game with logistic reaction functions are given in [5], where it is shown that two coexisting stable Nash equilibria are present. Their basins are multiply connected and non-connected sets, and the global (or contact) bifurcations which characterize their qualitative changes are generally revealed through computer assisted proofs, based on the detection of tangential contacts between different kinds of singularities, such as critical curves and repelling invariant curves. This is much more difficult for three-dimensional maps, since critical curves are replaced by critical surfaces, and the 

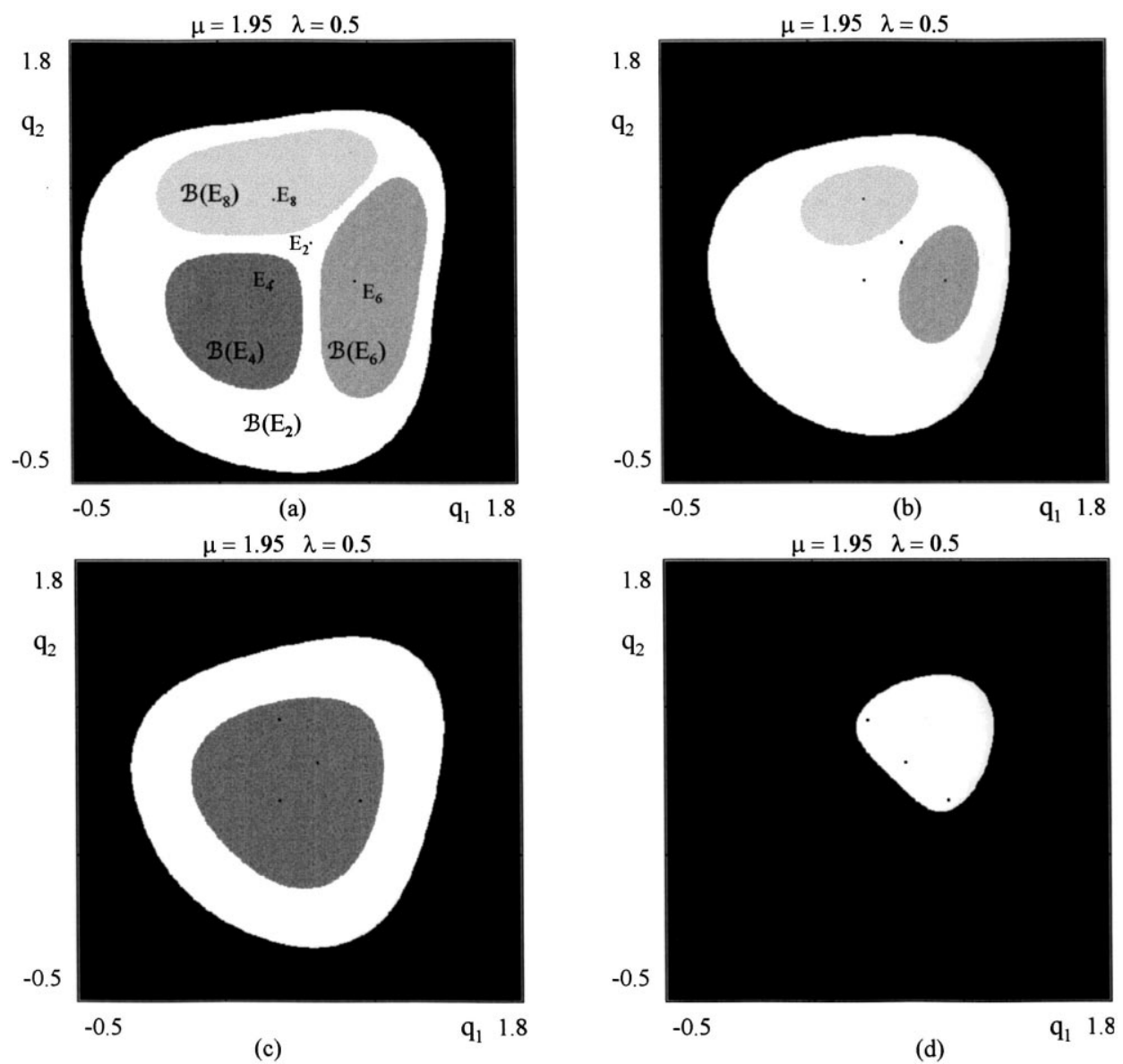

Fig. 2. Four different plane sections of the basins of the different attractors existing for $\mu=1.95$ and $\lambda=0.5$. The basins are represented by four different colors: the black region represents $\mathscr{B}(\infty)$, the white region represents $\mathscr{B}\left(E_{2}\right)$, the dark-grey region represents $\mathscr{B}\left(E_{4}\right)$, the intermediate-grey region represents $\mathscr{B}\left(E_{6}\right)$, the light-grey region represents $\mathscr{B}\left(E_{8}\right)$. (a) Plane section with the plane $q_{3}=1-1 / 2 \mu$. (b) Plane section with the plane $q_{3}=0$. (c) Plane section with the plane $q_{3}=1.2$. (d) Plane section with the plane $q_{3}=1.4$.

detection of the tangential contacts requires sophisticated usage of three-dimensional graphics. As a first modest step towards such results, in this paper we just give some numerical computations of the basins of coexisting attractors, and we show the occurrence of some qualitative changes in their structure.

Let us first consider a set of parameters in the darker region of Fig. 1 , namely $\mu=1.95$ and $\lambda=0.5$, for which we have four coexisting stable Nash equilibria, the stable nodes $E_{2}, E_{4}, E_{6}, E_{8}$. We try to understand the shape and the extension of the basins by taking planar sections of the three-dimensional basins. In Fig. 2 four different sections with planes $q_{3}=k$, i.e. parallel to the $\left(q_{1}, q_{2}\right)$ coordinate plane, are shown. The section shown in Fig. 2(a) is obtained by considering the plane with $q_{3}=1-1 / 2 \mu$, i.e., the plane through the fixed point $E_{2}$ and parallel to the $\left(q_{1}, q_{2}\right)$ coordinate plane. The different basins are represented by different colors: the black region represents the basin of infinity $\mathscr{B}(\infty)$, the white 

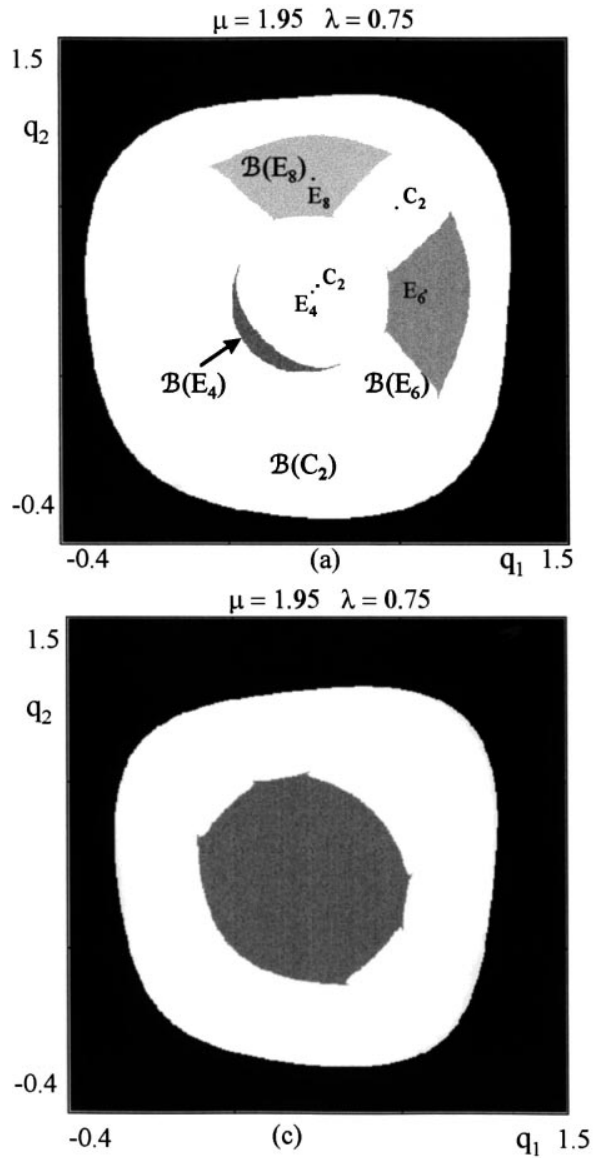

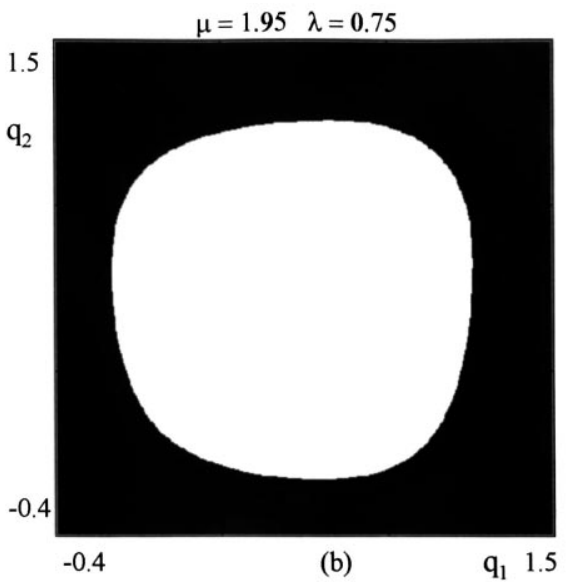

$\mu=1.95 \lambda=0.75$

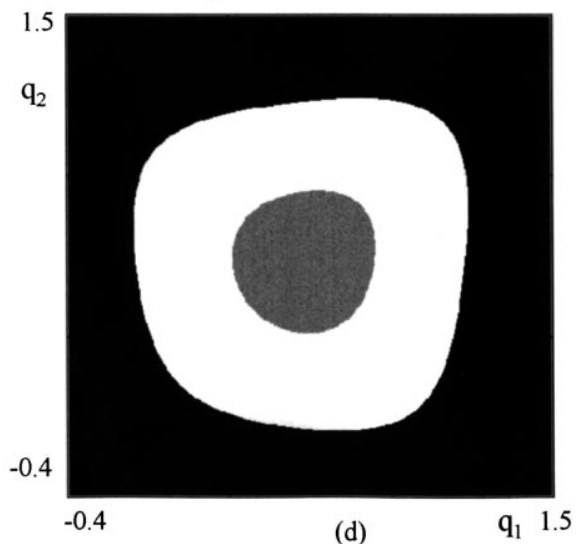

Fig. 3. Four different plane sections of the basins of the different attractors existing for $\mu=1.95$ and $\lambda=0.75$. The meaning of the different colors is the same as in Fig. 2. (a) Plane section with the plane $q_{3}=1-1 / 2 \mu$. (b) Plane section with the plane $q_{3}=0$. (c) Plane section with the plane $q_{3}=1$. (d) Plane section with the plane $q_{3}=1.1$.

region represents the basin of $E_{2}, \mathscr{B}\left(E_{2}\right)$, the dark-grey region represents the basin of $E_{4}, \mathscr{B}\left(E_{4}\right)$, the intermediate-grey region represents the basin of $E_{6}, \mathscr{B}(E 6)$, the light-grey region represents the basin of $E_{8}, \mathscr{B}\left(E_{8}\right)$. The black dots are the projections of the stable fixed points on the plane $q_{3}=k$.

In order to estimate the global three-dimensional shape of the basins, other sections with parallel planes $q_{3}=k$ are shown in Fig. 2. Fig. $2 \mathrm{~b}$ is obtained with the plane $q_{3}=0$. This figure shows that the basin $\mathscr{B}\left(E_{4}\right)$ has empty intersection with the plane $q_{3}=0$, i.e. the games starting with $q_{3}=0$ cannot converge to the Nash equilibrium $E_{4}$ (the equilibrium at which firm 3 dominates the market). Due to the symmetry, we can also deduce that the games starting with $q_{1}=0$ cannot converge to $E_{6}$ (the equilibrium at which firm 1 dominates the market) and those starting with $q_{2}=0$ cannot converge to $E_{8}$ (the equilibrium at which firm 2 dominates the market). Fig. $2 \mathrm{c}$ and $\mathrm{d}$ represent sections with the planes $q_{3}=1.2$ and $q_{3}=1.4$, respectively.

Fig. 3 was obtained with $\mu=1.95$ and $\lambda=0.75$. With these values of the parameters we have $\lambda(2 \mu-1)=2.175$, and besides the three stable Nash equilibria $E_{4}, E_{6}$ and $E_{8}$, a stable cycle of period 

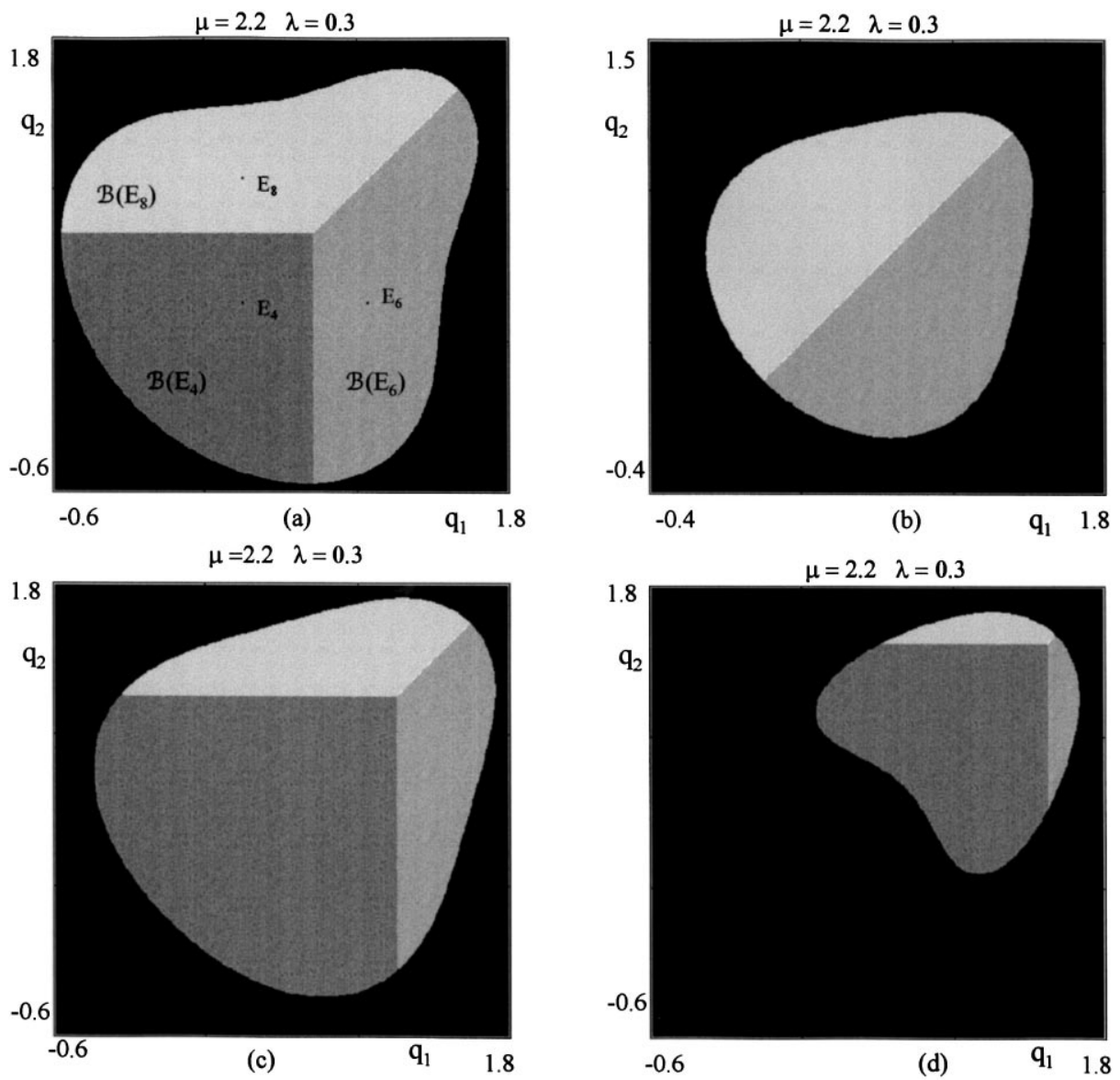

Fig. 4. Four different plane sections of the basins of the different attractors existing for $\mu=2.2$ and $\lambda=0.3$. The basins are represented by four different colors: the black region represents $\mathscr{B}(\infty)$, the dark-grey region represents $\mathscr{B}\left(E_{4}\right.$, the intermediate-grey region represents $\mathscr{B}\left(E_{6}\right)$, the light-grey region represents $\mathscr{B}\left(E_{8}\right)$. (a) Plane section with the plane $q_{3}=1-1 /$ $2 \mu$. (b) Plane section with the plane $q_{3}=0$. (c) Plane section with the plane $q_{3}=1.2$. (d) Plane section with the plane $q_{3}=1.5$.

2 exists, say $C_{2}=\{(0.86,0.86,0.86),(0.568,0.568,0.568)\} \in \Delta$, created by the flip bifurcation of $E_{2}$ occurring at $\lambda(2 \mu-1)=2$. Now the white region represents the basin $\mathscr{B}\left(C_{2}\right)$ of the 2-cycle, and the other colors have the same meaning as in Fig. 2. Fig. 3a represents the section with the plane $q_{3}=1-1 / 2 \mu$, and Fig. $3 \mathrm{~b}$ with the plane $q_{3}=0$, whereas Fig. $3 \mathrm{c}$ represents the section with the plane $q_{3}=1$ and Fig. $3 \mathrm{~d}$ with the plane $q_{3}=1$. . From these figures it seems that the smoothness of the basins boundaries has been lost. Additionally, they show that there is a large set of initial conditions for which the adjustment process fails to converge to a Nash equilibrium.

Fig. 4 was obtained for parameter values $\mu=2.2$ and $\lambda=0.3$, which belong to the region $\Omega_{2}^{\mathrm{s}}\left(E_{4}\right)$ of the stability domain $\Omega^{\mathrm{s}}\left(E_{4}\right)$. In this case the fixed points $E_{4}, E_{6}$ and $E_{8}$ are the only attractors, and $E_{2}$ is a saddle point. Besides the basin of infinity (black region) the set of points which generate bounded trajectories is shared by the three basins $\mathscr{B}\left(E_{4}\right), \mathscr{B}\left(E_{6}\right)$ and $\mathscr{B}\left(E_{8}\right)$, represented by the same colors as in the previous two figures. Also in this case, four different plane sections of the basins, with planes 


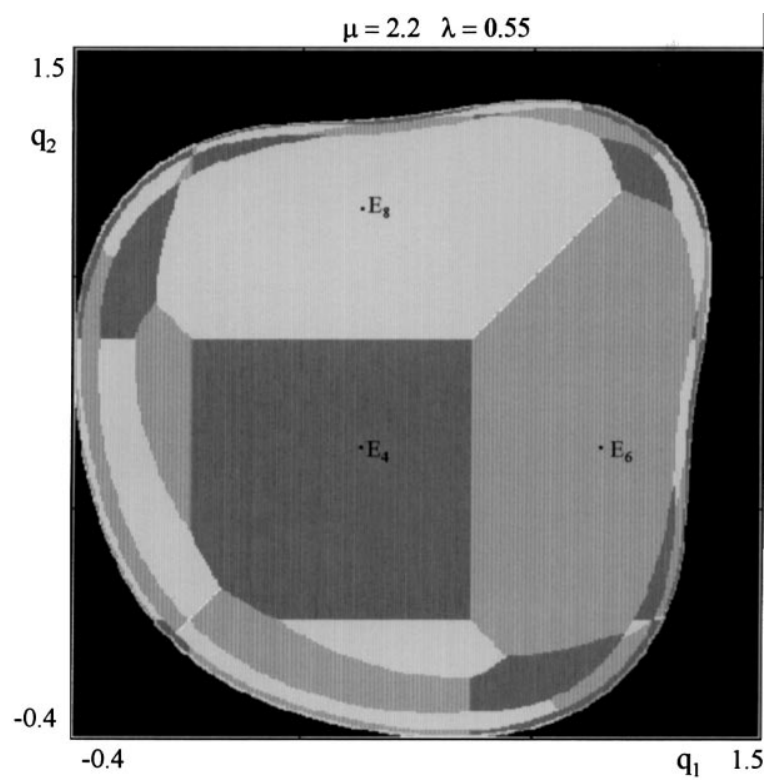

Fig. 5. Plane section, with the plane $q_{3}=0.7$, of the basins of the different attractors existing for $\mu=2.2$ and $\lambda=055$.

$q_{3}=k$, are shown: Fig. 4a was obtained with $k=1-1 / 2 \mu$, Fig. 4b with $k=0$, Fig. $4 \mathrm{c}$ with $k=1.2$, and Fig. $4 \mathrm{~d}$ with $k=1.5$. From these plane sections, all the basins appear to be connected subsets of $\mathbb{R}^{3}$. However, the situation drastically changes if higher values of the parameter $\lambda$ are considered. In fact, if $\lambda$ is increased, a global bifurcation occurs at which the basins of the three stable fixed points are transformed from simply connected sets into non-connected sets, each formed by many (really infinitely many) disjoint portions. For example, in Fig. 5, obtained for $\mu=2.2$ and $\lambda=0.5$, the plane section with the plane $q_{3}=0.7$ shows such a complex topological structure of the basins. We stress again that such a structure of the basins is peculiar of non-invertible maps, i.e. it cannot occur when the map is invertible.

In the case depicted in Fig. 5, smaller and smaller disjoint portions of all the three basins accumulate along the boundary $\partial \mathscr{B}(\infty)$. This is due to the fact that the boundary $\partial \mathscr{B}$, being a repelling set for the forward iteration of $T$, behaves as an attracting set for the iteration of the inverses of $T$.

The occurrence of global bifurcations which cause the transition from simple to complex basins structures, like the one described above, leads to a loss of predictability of the effect of small changes in the initial conditions. In fact, in the regions of phase space where many intermingled portions of different basins exist, a small displacement of the initial conditions, due, for example, to some exogenous perturbations which are always present in real systems, has a high probability of causing a crossing of some basin boundary and thus giving a different asymptotic evolution of the dynamical system.

\section{Complex attractors around the Nash equilibria}

When the Nash equilibria $E_{4}, E_{6}$ and $E_{8}$ lose stability through the supercritical Neimark-Hopf bifurcation, more complex attractors are created around them. This means that the long-run evolution of 


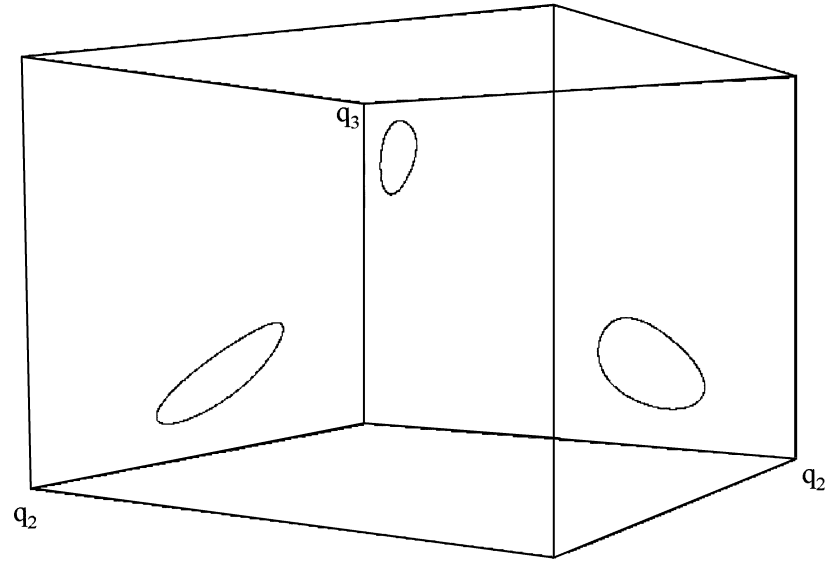

(a)

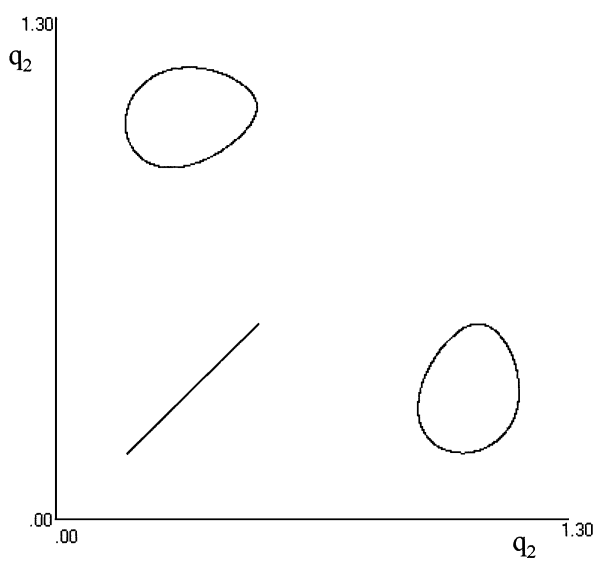

(b)

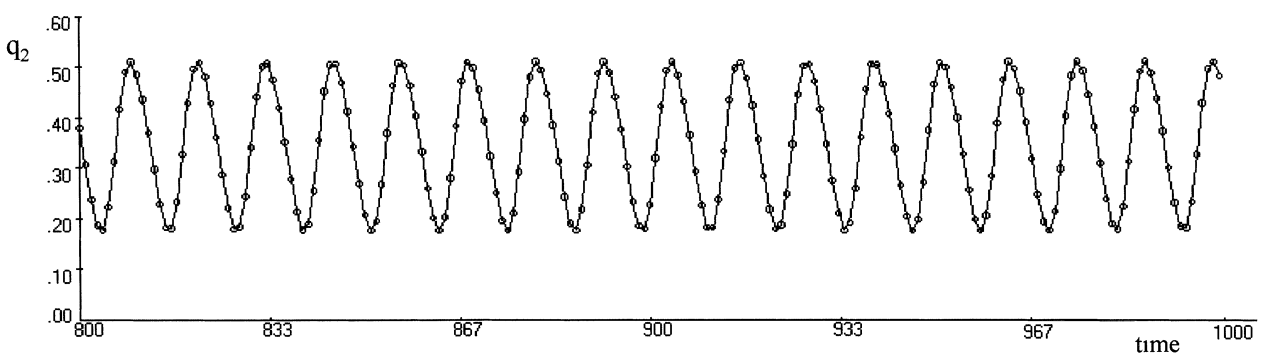

(c)

Fig. 6. For $\mu=2.5$ and $\lambda=0.25$, just after the Neimark-Hopf bifurcation, three trajectories are numerically computed starting from initial conditions close to the bifurcations fixed points $E_{4}, E_{6}$ and $E_{8}$, respectively. (a) Three-dimensional representation of the three trajectories (the early 300 points, representing the transient, are not plotted) (b) Projection of the trajectories on the plane $\left(q_{1}, q_{2}\right)$ (c) versus time representation of $q_{1}(t), t=800, \ldots, 1000$, for the trajectory starting close to $E_{4}$.

the oligopoly system is characterized by periodic or quasi-periodic fluctuations of production choices $q_{i}, i=1,2,3$, around the (locally) optimal values represented by the Nash equilibria. In other words, even if the adjustment process starts very close to a Nash equilibrium, the mechanism by which the competitors determine their production choices causes an asymptotic time evolution characterized by more or less regular oscillations, without converging to any optimal equilibrium point.

For example, let us consider values of the parameters $\lambda$ and $\mu$ taken just above the Neimark-Hopf bifurcation curve (see Fig. 1), such as $\mu=2.5$ and $\lambda=0.25>\lambda_{h}(2.5)=0.2$. With this set of parameters the three Nash equilibria $E_{4}, E_{6}$ and $E_{8}$ are unstable foci, with eigenvalues $z_{1}=0.52$ and $z_{2,3}=0.86 \pm 0.57 i$, with $\left|z_{2,3}\right|=1.03$. Since the three Neimark-Hopf bifurcations just occurred, we expect that three attracting closed invariant curves exist, on which the time evolution of the dynamical system is periodic or quasi periodic. The numerical iteration of the map Eq. (21) with $\mu=2.5$ and $\lambda=0.25$ confirms this, as shown in Fig. 6, where three numerically computed trajectories, obtained with initial conditions close to the fixed points $E_{4}, E_{6}$ and $E_{8}$, indicate the existence of three coexisting attractors. These are represented in three-dimensional phase space in Fig. 6a, and their projection on 
the $\left(q_{1}, q_{2}\right)$ coordinate plane is shown in Fig. 6b. Of course, the projections on the other two coordinate planes are identical, due to the symmetry properties of the iterated map. In Fig. 6c the values of $q_{1}(t)$, $t=800, \ldots, 1000$, obtained for the trajectory starting from an initial condition close to $E_{4}$, are plotted versus time. These numerical results suggests that a quasi-periodic motion occurs along each of the three closed invariant curves. Moreover, as stated at the end of Section 5, each attractor is embedded into the corresponding invariant plane, as it clearly appears from the projection shown in Fig. 6b. More precisely, the attractor around $E_{4}$ belongs to the invariant plane $\Pi_{3}$ of equation $q_{1}=q_{2}$, the one around $E_{6}$ belongs to the invariant plane $\Pi_{1}$ of equation $q_{2}=q_{3}$ and that around $E_{8}$ belongs to the invariant plane $\Pi_{2}$ of equation $q_{1}=q_{3}$. This means that the trajectories converging to such attractors are characterized by partial synchronization. Numerical explorations show that the basins of the three coexisting attractors are practically the same as those of the respective fixed points just before the occurrence of the bifurcation, i.e., they are similar to those shown in Fig. 4.

As the parameters move away from the Hopf bifurcation curve, in the region where no stable Nash equilibria exist, the amplitude of the three stable closed orbits increases, then they are transformed into more and more complex attractors. For example, with $\mu=2.5$ and $\lambda=0.48$ the trajectories starting close to the fixed points $E_{i}, i=4,6,8$, are attracted to three distinct chaotic attractors, as shown in

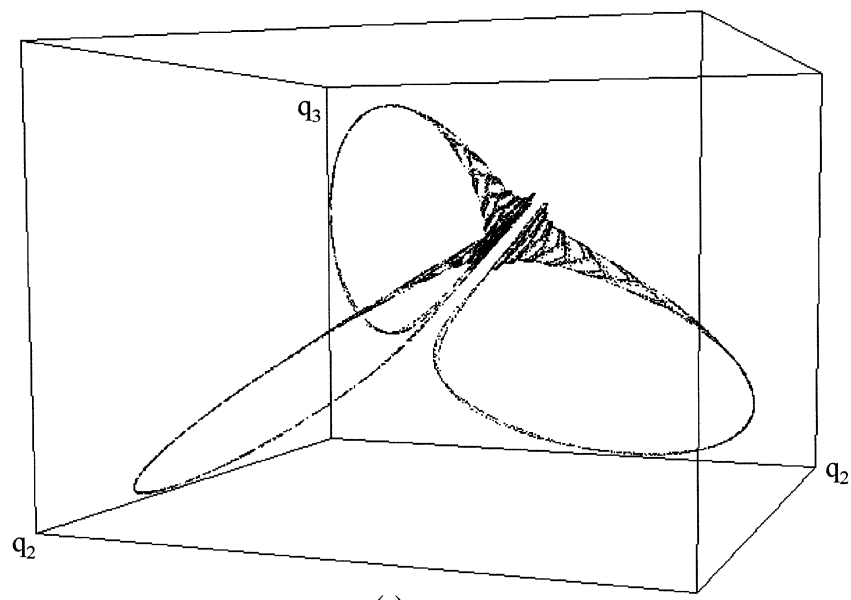

(a)

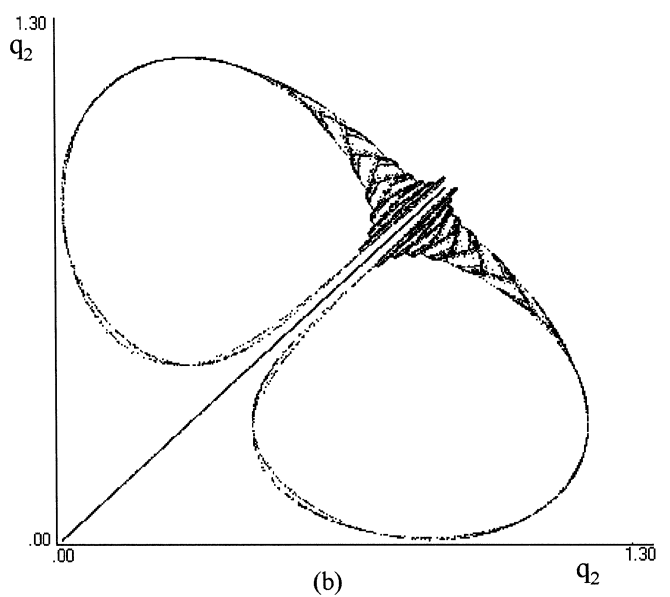

(b)

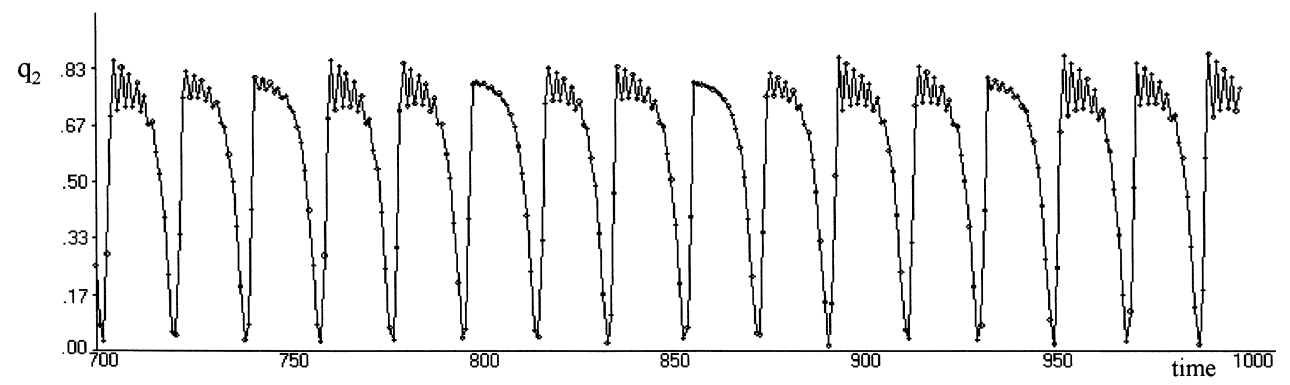

(c)

Fig. 7. For $\mu=2.5$ and $\lambda=0.48$, three trajectories are numerically computed starting from initial conditions close to the fixed points $E_{4}, E_{6}$ and $E_{8}$, respectively. (a) Three-dimensional representation of the three trajectories (the early 300 points, representing the transient, are not plotted). (b) Projection of the trajectories on the plane $\left(q_{1}, q_{2}\right)$. (c) Versus time representation of $q_{1}(t), t=800, \ldots, 1000$, for the trajectory starting close to $E_{4}$. 
Fig. 7. Also in this case, the projections of the chaotic attractors on the coordinate planes clearly show that each attractor is embedded into the corresponding invariant plane, i.e. the trajectories converging to them evolve toward situations of partial synchronization, even if they are chaotic trajectories. The time evolution of $q_{1}(t)$ along the trajectory embedded into $\Pi_{3}$ is represented in Fig. 7c.

As the parameters $\mu$ and $\lambda$ are further moved away from the Neimark-Hopf bifurcation curve the three chaotic attractors disappear due to a contact with the boundary of their immediate basins. Such a contact causes the destruction of the chaotic attractors, i.e., they become chaotic repellors after the contact (see [13,14]), a situation called final bifurcation in [1,17] or boundary crisis in [15].

\section{Numerical simulations in the case of heterogeneous players}

We assume now that Eq. (10) still holds, but relax Eq. (20). That is, the competitors exhibit heterogeneous behavior, due to different speeds of adjustment. In this case the symmetry properties described in Section 4, and the consequent existence of invariant submanifolds of partial synchronization $\Pi_{i}, i=1,2,3$ and of total synchronization $\Delta$, no longer hold.

The fixed points are the same as those computed in Section 2, because they do not depend on the parameters $\lambda_{i}$. However, in the case of heterogeneous behavior it is not so easy to obtain analytical expressions of the local bifurcations conditions, and the consequent delimitation of the stability regions, in the four-dimensional space of parameters:

$$
\Omega_{4}=\left\{\left(\mu, \lambda_{1}, \lambda_{2}, \lambda_{3}\right) \mid \mu>0,0 \leq \lambda_{i} \leq 1, \quad i=1,2,3\right\} .
$$

Of course, the stability regions in $\Omega_{4}$ must be such that their intersections with the two-dimensional submanifold of $\Omega_{4}$ defined by the two equations $\lambda_{1}=\lambda_{2}$ and $\lambda_{2}=\lambda_{3}$ give the corresponding stability regions for the homogeneous case as described in Proposition 1. So, the case of heterogeneous competitors can be studied starting from the benchmark case of the homogeneous case by introducing small differences between the speeds of adjustment. For example, starting from the situation shown in Fig. 2, obtained with $\mu=1.95$ and $\lambda_{1}=\lambda_{2}=\lambda_{3}=0.5$, and characterized by the presence of the four coexisting stable fixed points $E_{i}, i=2,4,6,8$, we introduce different speeds of adjustment by decreasing $\lambda_{1}$ and increasing $\lambda_{3}$, namely $\lambda_{1}=0.3, \lambda_{2}=0.5, \lambda_{3}=0.7$. The numerical computation of the eigenvalues shows that the four fixed points remain stable nodes, being:

$$
\begin{array}{lllrl}
\lambda_{1}\left(E_{2}\right)=0.982 \ldots, & \lambda_{2}\left(E_{2}\right)=-0.453 \ldots, & & \lambda_{3}\left(E_{2}\right)=0.974 \ldots, \\
\lambda_{1}\left(E_{4}\right)=0.867 \ldots, & \lambda_{2}\left(E_{4}\right)=-0.040 \ldots, & & \lambda_{3}\left(E_{4}\right)=0.673 \ldots, \\
\lambda_{1}\left(E_{6}\right)=0.896 \ldots, & \lambda_{2}\left(E_{6}\right)=0.075 \ldots, & \lambda_{3}\left(E_{6}\right)=0.528 \ldots, \\
\lambda_{1}\left(E_{8}\right)=0.878 \ldots, & \lambda_{2}\left(E_{8}\right)=-0.01 \ldots, & \lambda_{3}\left(E_{8}\right)=0.632 \ldots,
\end{array}
$$

We observe that the introduced heterogeneity in the speeds of adjustment does not seem to have a large influence on the eigenvalues of the fixed points (it is easy to compare the eigenvalues given above with those obtained with the set of parameters used in Fig. 2, which can be computed by their analytical expression given in Section 5). However, a remarkable change occurs in the basins, as can be immediately deduced from Fig. 8a, where the section of the basins with the plane of equation $q_{3}=1-1 / 2 \mu$ is shown (the meaning of the colors is the same as in Fig. 2). From a comparison between 


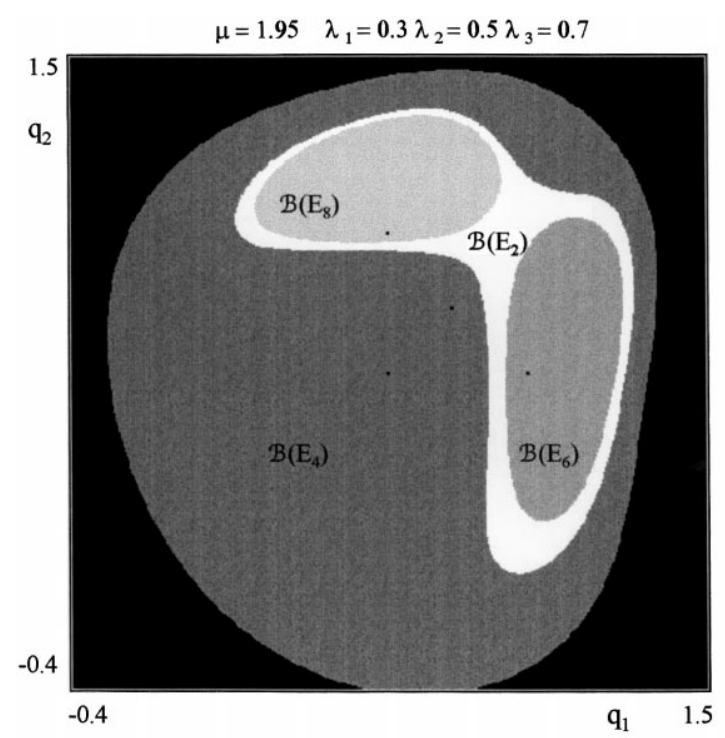

(a)

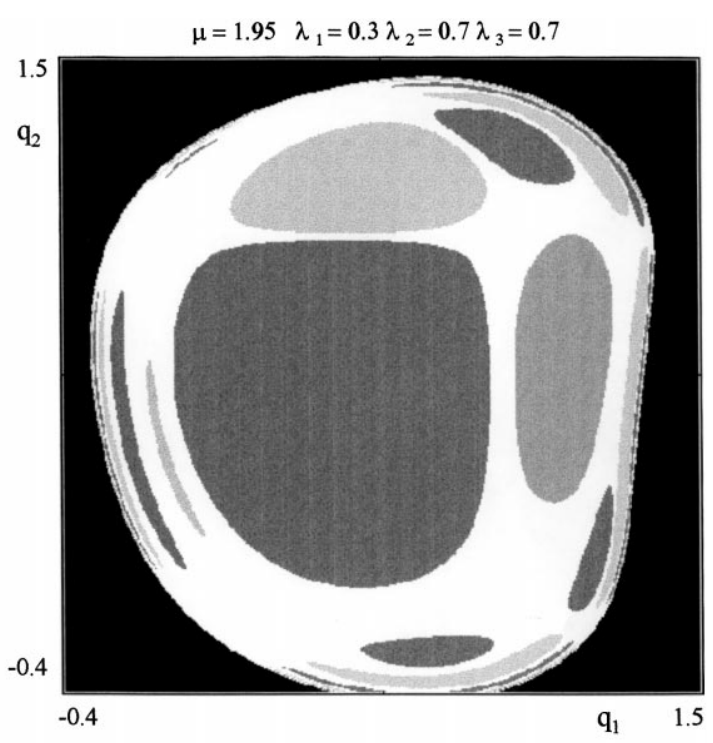

(b)

Fig. 8. Plane section with the plane $q_{3}=0.7$ of the basins of the attractors existing in the case of heterogeneous firms with $\mu=1.95$ and with (a) $\lambda_{1}=0.3, \lambda_{2}=0.5, \lambda_{3}=0.7$. (b) $\lambda_{1}=0.3, \lambda_{2}=0.7, \lambda_{3}=0.7$.

Fig. 8a and Fig. 2a it is obvious that the introduction of a difference in the speeds of adjustment causes a noticeable enlargement of $\mathscr{B}\left(E_{4}\right)$. We recall that, among the stable fixed points, $E_{4}$ is the one with a larger value of $q_{3}$, i.e. it is characterized by a dominance of firm 3. So, we may conjecture that an increase in $\lambda_{3}$, the speed of adjustment of firm 3, causes an enlargement of the basin of the stable equilibrium where firm 3 dominates the market. Indeed, this conjecture seems to be confirmed by other numerical simulations, in the sense that if $\lambda_{3}$ is decreased and $\lambda_{1}$ is increased then the basin of $E_{6}$ (the stable equilibrium where firm 1 dominates) becomes larger, an so on. Of course, a greater extension of the basin means a higher probability of convergence to the corresponding equilibrium, or a greater robustness of the stable equilibrium with respect to exogenous shocks.

The effect of heterogeneity with regard to the speeds of adjustment on the structure of the basins is even more evident in the situation shown in Fig. 8b, where the same plane section as in Fig. 8a is shown, obtained with $\lambda_{1}=0.3, \lambda_{2}=0.7, \lambda_{3}=0.7$, i.e. after an increase of $\lambda_{2}$ with respect to the value used in Fig. 8a. In this case the topological structure of the basins $\mathscr{B}\left(E_{4}\right)$ and $\mathscr{B}\left(E_{8}\right)$ is much more complex, because now these two basins are non-connected sets, formed by infinitely many disjoint portions which accumulate along the boundary $\partial \mathscr{B}(\infty)$. In contrast, $\mathscr{B}\left(E_{6}\right)$ remains simply connected. We remark that $E_{6}$ is the stable steady state characterized by the highest value of $q_{1}$, the output of the firm with smallest speed of adjustment. As already stressed in Section 6, increasing values of the speeds of adjustment may cause the occurrence of a global bifurcation at which the basins are transformed from simply connected to non-connected sets. In the case of heterogenous behavior this may happen when at least one speed of adjustment is increased, and the change of the topological structure does not necessarily involve all the basins.

To sum up, we can say that the heterogeneity in the firms' adjustment speeds does not have a great influence on the local stability properties, related to the modulus of the eigenvalues, but may have a 
remarkable effect on structure of the basins. In particular, a sort of heterogeneity in the basins structure is induced by introducing differences in the behavior of firms.

The coexistence of several attractors is numerically observed also in the presence of attractors which are more complex than steady states. In order to show this, we again start from the benchmark case of homogeneous competitors, studied in Section 7, and we introduce small changes in the speeds of adjustment. For example, starting from the set of parameters used in Fig. 7, i.e. $\mu=2.5, \lambda_{1}=\lambda_{2}=$ $\lambda_{3}=0.48$, we consider the situation obtained with the same value of $\mu$ and with $\lambda_{1}=0.4, \lambda_{2}=0.48$, $\lambda_{3}=0.5$. With this set of parameters we generate three trajectories, starting from initial conditions close to the unstable foci $E_{4}, E_{6}$ and $E_{8}$, respectively. The result of this numerical simulation is shown in Fig. 9, where three coexisting attractors are seen, each located around one of the fixed points, but now the invariant planes no longer exist: the trajectory starting from the initial condition close to $E_{4}$ converges to a chaotic attractor, the trajectory starting from the initial condition close to $E_{6}$ converges to another chaotic attractor and the trajectory starting from the initial condition close to $E_{8}$ converges to a cycle of period 43. The projections of these three attractors on the plane $\left(q_{1}, q_{2}\right)$ are shown in Fig. $9 \mathrm{~b}$ and the corresponding representations of $q_{1}(t)$ versus time along these trajectories are shown in Fig. 9c for $t \in[800,1000]$. In Fig. 9b we can see that, differently from the homogenous case, the three attractors are not embedded into the partial synchronization planes, i.e. they are truly three-dimensional attractors.

Another consequence of the symmetry breaking due to the introduction of heterogeneities is that the disappearance of the attractors, caused by contacts with the boundary of their immediate basins, is not simultaneous, due to the asymmetry of the basins induced by the asymmetry of the map Eq. (5). For example, if we introduce a small increase of $\lambda_{3}$ with respect to the value used to obtain Fig. 9, the attractor around $E_{6}$ no longer exists. This is shown in Fig. 10, where three trajectories are numerically generated as in Fig. 9, but those starting close to $E_{4}$ and $E_{6}$ converge to the same attractor, located around $E_{4}$.

\section{Conclusions}

In this paper we introduced an oligopoly model with three competitors, and we assumed that firms determine their current quantities by a partial adjustment to the Best Response. The particular form of the reaction functions of the firms can be derived by assuming that some kind of interfirm externality exists among the firms, i.e. the corresponding (marginal) payoffs of the firms are (positively) influenced by the actions of other firms. We analyzed the resulting time-discrete model and we derived stability conditions for the Nash equilibria (fixed points of the map) in the case of homogeneous firms. We showed that for various parameter constellations several coexisting Nash equilibria or more complex attractors emerge. In particular, we tried to emphasize the importance of a global analysis, which stresses the role of initial conditions on the long run outcome of the market. In other words, our focus has been on the set of initially chosen quantities which converge to one of several coexisting attractors and the changes these sets undergo as parameters are varied.

The conclusions which can be derived from our analysis relate to the insights gained for economists and for mathematicians. For researchers in the field of economics, the coexistence of several Nash equilibria poses a problem, since in this case the considered game does not have a unique prediction how it will be played. Even the stability properties of the Nash equilibria can not be used as a proper refinement of the definition of an equilibrium. If the basins are simply connected, the extent of the 


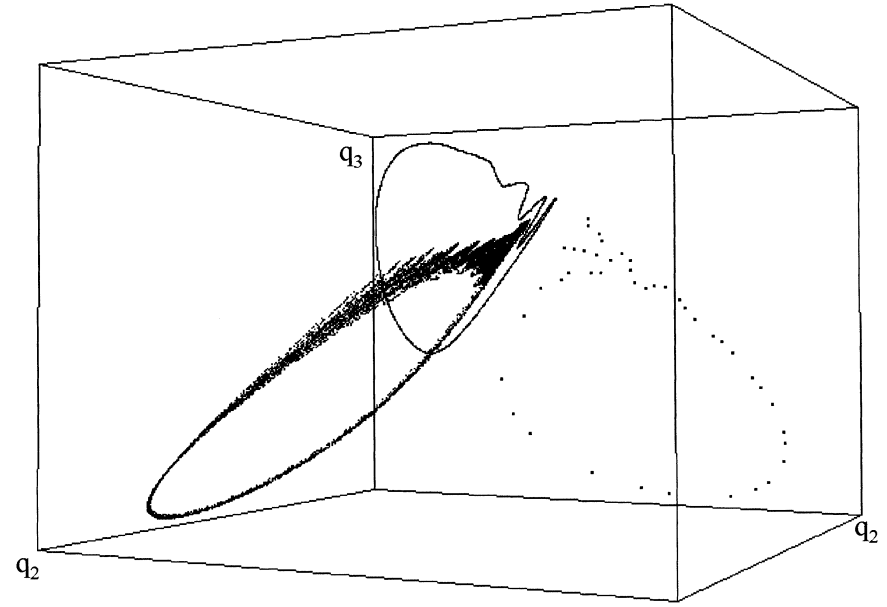

(a)

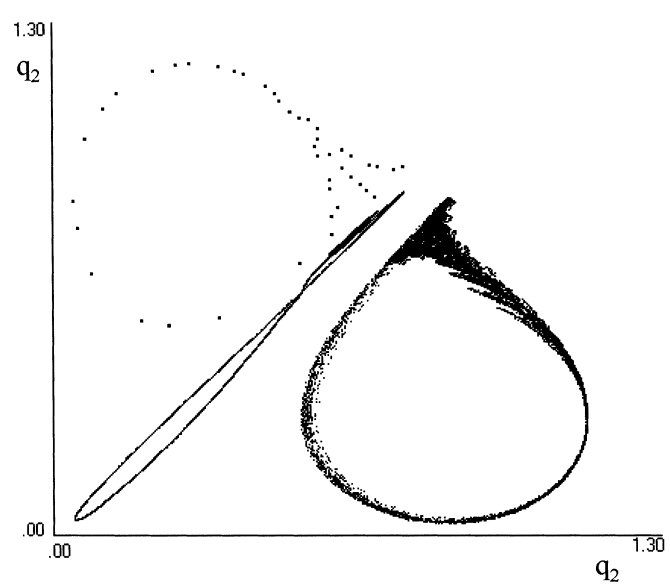

(b)
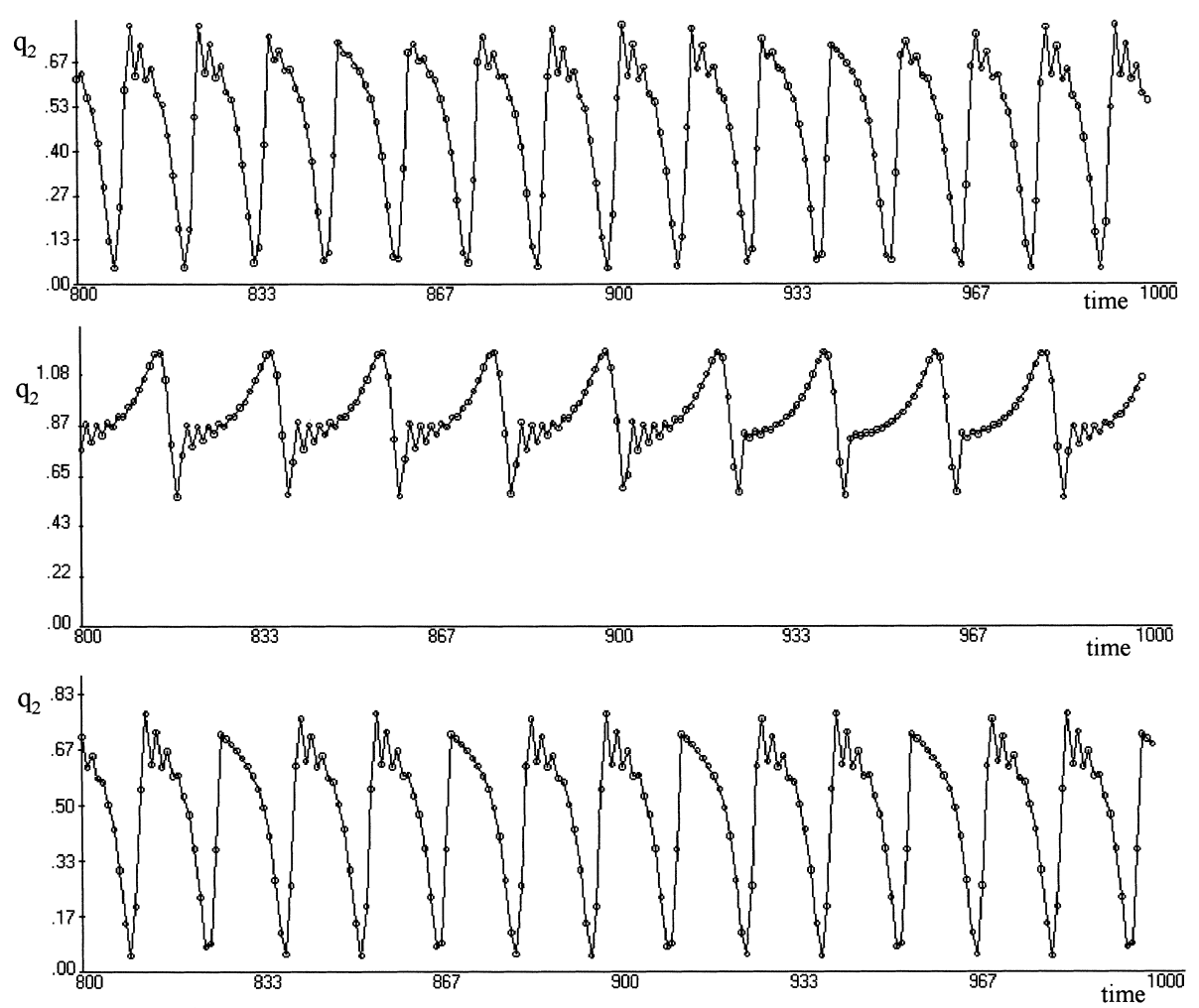

(c)

Fig. 9. For $\mu=2.5, \lambda_{1}=0.4, \lambda_{2}=0.48$ and $\lambda_{3}=0.5$ three trajectories are numerically computed starting from initial conditions close to the fixed points $E_{4}, E_{6}$ and $E_{8}$, respectively. (a) Three-dimensional representation of the three trajectories (the early 300 points, representing the transient, are not plotted). (b) Projection of the trajectories on the plane $\left(q_{1}, q_{2}\right)$. (c) Versus time representations of $q_{1}(t), t=800, \ldots, 1000$, for the trajectories starting close to $E_{4}, E_{6}$ and $E_{8}$, respectively. 


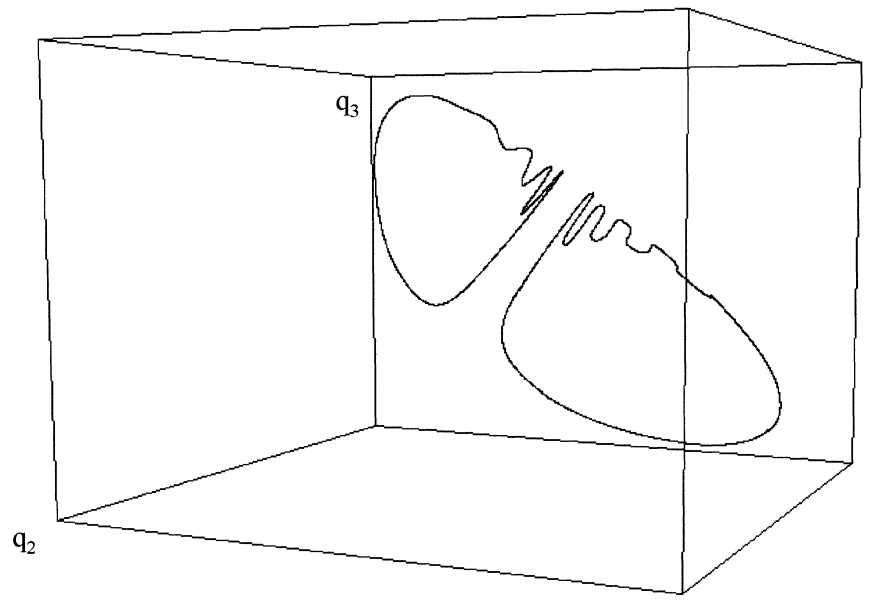

(a)

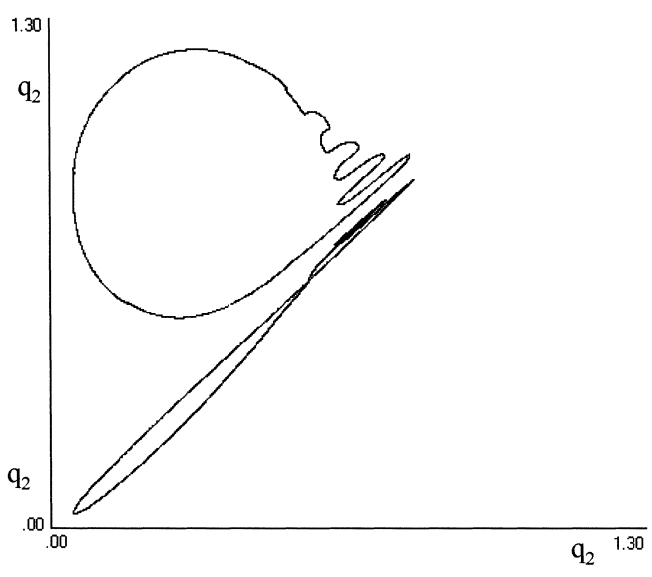

(b)

Fig. 10. For $\mu=2.5, \lambda_{1}=0.4, \lambda_{2}=0.48$ and $\lambda_{3}=0.505$ three trajectories are numerically computed starting from initial conditions close to the fixed points $E_{4}, E_{6}$ and $E_{8}$, respectively. Two of them, those starting close to $E_{4}$ and $E_{6}$, converge to the same attractor, located around $E_{4}$. (a) Three-dimensional representation of the three trajectories (the early 300 points, representing the transient, are not plotted). (b) Projection of the trajectories on the plane $\left(q_{1}, q_{2}\right)$.

basins of these Nash equilibria might at least serve as a measure of the probability or plausibility of a particular prediction. Furthermore, it might serve as a measure of the robustness of the model under investigation with regard to exogenous perturbations. However, the result that despite the local stability of an equilibrium $N E_{1}$, the adjustment process might lead to a different equilibrium $N E_{2}$, even if the initially chosen quantities are (relatively) close to $N E_{1}$ (in the case of intermingled basins) is surprising and puzzling for applied economists. It demonstrates that the predictions of the model are not robust with respect to perturbations in the initial quantities, and makes us aware that in non-linear models global properties of the equilibria have to be studied and these particular parameter regions have to be identified.

From a mathematical point of view our analysis provided a (partial) characterization of the properties of a three-dimensional discrete-time dynamical system which exhibits rather complex behavior. We have demonstrated that this complexity stems from both the creation of complex attractors in the three dimensional phase space and the coexistence of attracting sets with rather complicated basin boundaries. As pointed out before, the mathematical treatment of three-dimensional non-invertible maps is an almost unexplored field, and our investigation may be seen as a step in this direction. Finally, as we assumed strong symmetry properties of the map, our analytical results are specific to this situation, but may nevertheless constitute a good starting point for a more complete study of the global properties and bifurcations of the complex attractors and their basins in higher-dimensional noninvertible maps.

\section{Acknowledgements}

We thank Laura Gardini for helpful comments and Paolo Tenti for the three-dimensional graphical programs. The paper has been written while M. Kopel was visiting the University of Constance, Faculty 
of Economics and Statistics, Germany. The hospitality of the department is gratefully acknowledged. This work has been performed under the auspices of CNR, Italy, and under the activity of the national research project 'Nonlinear Dynamics and Stochastic Models in Economics and Finance', MURST, Italy.

\section{References}

[1] R. Abraham, L. Gardini, C. Mira, Chaos in Discrete Dynamical Systems (A Visual Introduction in Two Dimension) Springer, Berlin, 1997.

[2] H.N. Agiza, On the analysis of stability, bifurcation, chaos and chaos control of Kopel map, Chaos, Solitons \& Fractals, in press.

[3] E. Ahmed, H.N. Agiza, Dynamics of a Cournot game with $n$ competitors, Chaos, Solitons \& Fractals 9 (1998) 15131517.

[4] G.I. Bischi, A. Naimzada, Global analysis of a dynamic duopoly game with bounded rationality, in: Advances in Dynamic Games and Applications, vol. 5, Birkhauser, Boston, in press.

[5] G.I. Bischi, M. Kopel, On the basins of multiple Nash equilibria in nonlinear Duopoly games, submitted for publication.

[6] G.I Bischi, C. Mammana, L. Gardini, Multistability and cyclic attractors in duopoly games, Chaos, Solitons \& Fractals, in press.

[7] G.I. Bischi, L. Stefanini, L. Gardini, Synchronization, intermittency and critical curves in a duopoly game, Math. Computers in Simulation 44 (1998) 559-585.

[8] P. Chossat, M. Golubitsky, Iterates of maps with symmetry, SIAM J. Math. Anal. 19 (1988) 1259-1270.

[9] A. Cournot, Researches Into the Principles of Wealth, 1963 (English Translation), Irwin Paperback Classics in Economics, 1938.

[10] J. Friedman, Oligopoly theory, in: K.J. Arrow, M.D. Intriligator (Eds.), Handbook of Mathematical Economics, vol. II, North-Holland, Amsterdam, 1982.

[11] L. Gardini, R. Abraham, R. Record, D. Fournier-Prunaret, A double logistic map, Int. J. Bifurcations Chaos 4(1) (1994) 145-176.

[12] J. Guckenheimer, P. Holmes. Nonlinear Oscillations, Dynamical Systems, and Bifurcations of Vector Fields, Springer, Berlin, 1983.

[13] I. Gumowski, C. Mira, Bifurcation déstabilisant une solution chaotique d'un endomorphisme du 2nd ordre, Comptes Rendus Acad. Sci. Paris, Série A 286 (1978) 427-431.

[14] I. Gumowski, C. Mira, Dynamique, Chaotique Cepadues Editions, Toulose, 1980.

[15] C. Grebogi, E. Ott, J.A. Yorke, Crises, sudden changes in chaotic attractors and transient chaos, Physica D 7 (1983) 181200.

[16] M. Kopel, Simple and complex adjustment dynamics in Cournot duopoly models, Chaos, Solitons \& Fractals 7 (1996) 2031-2048.

[17] C. Mira, L. Gardini, A. Barugola, J.C. Cathala, Chaotic dynamics, in: Two-Dimensional Noninvertible Maps, World Scientific, Singapore, 1996.

[18] C. Mira, D. Fournier-Prunaret, L. Gardini, H. Kawakami, J.C. Cathala, Basin bifurcations of two-dimensional noninvertible maps: fractalization of basins, Int. J. Bifurcation Chaos 4 (1994) 343-381.

[19] T. Puu, Chaos in duopoly pricing, Chaos, Solitons \& Fractals 1 (1991) 573-581.

[20] T. Puu, Complex dynamics with three oligopolists, Chaos, Solitons \& Fractals 7 (1996) 2075-2081.

[21] T. Puu, The chaotic duopolists revisited, J. Econ. Behaviour Org. 33 (1998) 385-394.

[22] D. Rand, Exotic phenomena in games and duopoly models, J. Math. Econ. 5 (1978) 173-184. 